\title{
Bernoulli numbers, convolution sums and congruences of coefficients for certain generating functions
}

Daeyeoul Kim ${ }^{1 *}$, Aeran Kim² and Ayyadurai Sankaranarayanan ${ }^{3,4}$

\section{"Correspondence:}

daeyeoul@nims.re.kr

${ }^{1}$ National Institute for Mathematical

Sciences, Yuseong-daero 1689-gil,

Yuseong-gu, Daejeon, 305-811,

South Korea

Full list of author information is

available at the end of the article

\begin{abstract}
In this paper, we study the convolution sums involving restricted divisor functions, their generalizations, their relations to Bernoulli numbers, and some interesting applications.

MSC: 11B68; 11A25; 11A67; 11Y70; 33E99
\end{abstract}

Keywords: Bernoulli numbers; generating functions; divisor functions; convolution sums

\section{Introduction}

The Bernoulli polynomials $B_{k}(x)$, which are usually defined by the exponential generating function

$$
\frac{t e^{x t}}{e^{t}-1}=\sum_{k=0}^{\infty} B_{k}(x) \frac{t^{k}}{k !}
$$

play an important role in different areas of mathematics, including number theory and the theory of finite differences. The Bernoulli polynomials satisfy the following well-known identities :

$$
\begin{aligned}
& B_{k}(x+1)-B_{k}(x)=k x^{k-1}, \quad k \geq 1, \\
& \frac{d}{d x} B_{k}(x)=k B_{k-1}(x), \quad k \geq 1,
\end{aligned}
$$

and

$$
\sum_{j=0}^{N} j^{k}=\frac{B_{k+1}(N+1)-B_{k+1}(0)}{k+1}, \quad k \geq 1 .
$$

We set $B_{k}=B_{k}(0)$. It is obvious from the way the polynomials $B_{k}(x)$ are constructed that all the $B_{k}$ are rational numbers. It can be shown that $B_{2 k+1}=0$ for $k \geq 1$, and is alternatively positive and negative for even $k$. The $B_{k}$ are called Bernoulli numbers.

() 2013 Kim et al.; licensee Springer. This is an Open Access article distributed under the terms of the Creative Commons Attribution License (http://creativecommons.org/licenses/by/2.0), which permits unrestricted use, distribution, and reproduction in any medium, provided the original work is properly cited. 
Throughout the paper, we use the following arithmetical functions and $q$-series (sometimes defined by product expressions). For any integer $N \geq 1, l, s \in \mathbb{N} \cup\{0\}$, we define

$$
\begin{aligned}
& \sigma_{l}(N):=\sum_{d \mid N} d^{l}, \quad \sigma_{s}^{*}(N ; p):=\sum_{\substack{d \mid N \\
\frac{N}{d} \neq 0(\bmod p)}} d^{s}, \quad \sigma_{s}^{*}(N):=\sigma_{s}^{*}(N ; 2)=\sum_{\substack{d \mid N \\
N / d \text { odd }}} d^{s}, \\
& \widetilde{\sigma}_{s}(N):=\sum_{d \mid N}(-1)^{d-1} d^{s}, \quad S_{i}(N):=\sum_{k=1}^{N-1} k^{i} .
\end{aligned}
$$

For $q \in \mathbb{C}$ with $|q|<1$, we consider the $q$-series:

$$
\begin{aligned}
& \mathcal{A}(q):=\sum_{N=1}^{\infty} \sigma_{1}^{*}(N) q^{N}, \quad \mathcal{B}(q):=\sum_{N=1}^{\infty} \sigma_{3}^{*}(N) q^{N}, \quad \mathcal{C}(q):=\sum_{N=1}^{\infty} \sigma_{5}^{*}(N) q^{N}, \\
& \sum_{N=1}^{\infty} a(N) q^{N}:=q \prod_{N=1}^{\infty}\left(1-q^{N}\right)^{2}\left(1-q^{2 N}\right)^{2}\left(1-q^{3 N}\right)^{2}\left(1-q^{6 N}\right)^{2}, \\
& \sum_{N=1}^{\infty} b(N) q^{N}:=q \prod_{N=1}^{\infty}\left(1-q^{N}\right)^{8}\left(1-q^{2 N}\right)^{8}, \\
& \sum_{N=1}^{\infty} c(N) q^{N}:=q \prod_{N=1}^{\infty}\left(1-q^{N}\right)^{16}\left(1-q^{2 N}\right)^{4}, \quad \sum_{N=1}^{\infty} \tau(N) q^{N}:=q \prod_{N=1}^{\infty}\left(1-q^{N}\right)^{24}, \\
& \sum_{N=1}^{\infty} l(N) q^{N}:=q^{2} \prod_{N=1}^{\infty}\left(1-q^{N}\right)^{8}\left(1-q^{2 N}\right)^{4}\left(1-q^{4 N}\right)^{8} .
\end{aligned}
$$

The exact evaluation of the basic convolution sum

$$
\sum_{k=1}^{N-1} \sigma_{1}(k) \sigma_{1}(N-k)
$$

first appeared in a letter from Besge to Liouville in 1862. The evaluation of such sums also appear in the works of Glaisher, Lahiri, Lehmer, Ramanujan, and Skoruppa. For instance, Ramanujan [1] obtained

$$
\begin{aligned}
& \sum_{k=1}^{N-1} \sigma_{1}(k) \sigma_{1}(N-k)=\frac{1}{12}\left(5 \sigma_{3}(N)+(1-6 N) \sigma(N)\right) \text { and } \\
& \sum_{k=1}^{N-1} \sigma_{1}(k) \sigma_{3}(N-k)=\frac{1}{240}\left[21 \sigma_{5}(N)+(10-30 N) \sigma_{3}(N)-\sigma_{1}(N)\right]
\end{aligned}
$$

using only elementary arguments. For $a, b, N \in \mathbb{N}$, Ramanujan showed that the sum

$$
S_{a, b}(N):=\sum_{m=1}^{N-1} \sigma_{a}(m) \sigma_{b}(N-m)
$$

can be evaluated in terms of the quantities

$$
\sigma_{a+b+1}(N), \quad \sigma_{a+b-1}(N), \quad \ldots, \quad \sigma_{3}(N), \quad \sigma_{1}(N)
$$


for the nine pairs $(a, b) \in \mathbb{N}^{2}$ satisfying

$$
a+b=2,4,6,8,12, \quad a \leq b, a \equiv b \equiv 1 \quad(\bmod 2) .
$$

For explicit evaluations of $S_{a, b}(N)$ for different pairs $(a, b) \in \mathbb{N}^{2}$ satisfying the above conditions stated, we refer to the papers of Ramanujan [1], [2], Huard et al. [3], Lahiri [4], and Glaisher [5], respectively. Levit [6] showed that the nine arithmetic evaluations of $S_{a, b}(N)$ (with $a, b$ both odd) are the only ones using the theory of modular forms. In [4], Lahiri has given 37 sums of the form

$$
\sum_{\substack{\left(m_{1}, \ldots, m_{r}\right) \\ m_{1}+\cdots+n_{r}=N}} m_{1}^{a_{1}} \cdots m_{r}^{a_{r}} \sigma_{b_{1}}\left(m_{1}\right) \cdots \sigma_{b_{r}}\left(m_{r}\right)
$$

where $a_{1}, \ldots, a_{r} \in \mathbb{N}_{0}:=\mathbb{N} \cup\{0\}, b_{1}, \ldots, b_{r} \in \mathbb{N}$, each of which can be expressed as a finite linear combination of $\sigma_{1}(N), \sigma_{3}(N), \ldots, \sigma_{b_{1}+b_{2}+\cdots+b_{r}+r-1}(N)$ with coefficients which are polynomials in $N$ of degree at most $a_{1}+\cdots+a_{r}+r-1$ with rational coefficients.

In 2002, Huard et al. [3] extended Melfi's [7] result to

$$
\begin{aligned}
& \sum_{k<N / 2} \sigma_{1}(k) \sigma_{3}(N-2 k) \\
& \quad=\frac{1}{48} \sigma_{5}(N)+\frac{1}{15} \sigma_{5}\left(\frac{N}{2}\right)+\frac{(2-3 N)}{48} \sigma_{3}(N)-\frac{1}{240} \sigma_{1}\left(\frac{N}{2}\right), \\
& \sum_{k<N / 2} \sigma_{3}(k) \sigma_{1}(N-2 k) \\
& \quad=\frac{1}{240} \sigma_{5}(N)+\frac{1}{12} \sigma_{5}\left(\frac{N}{2}\right)+\frac{(1-3 N)}{24} \sigma_{3}\left(\frac{N}{2}\right)-\frac{1}{240} \sigma_{1}(N),
\end{aligned}
$$

where $N$ is an arbitrary positive integer.

Glaisher $[5,8,9]$ extended Besge's formula by replacing $\sigma_{1}(N)$ in the convolution sum in (1) by other arithmetical functions; for example, he obtained

$$
\begin{aligned}
24 \sum_{k=1}^{N-1} \sigma_{1}^{*}(k) \sigma_{1}^{*}(N-k) & =6 \sigma_{3}(N)-6 \sigma_{3}\left(\frac{N}{2}\right)-6 N \sigma_{1}(N)+6 N \sigma_{1}\left(\frac{N}{2}\right) \\
& =6\left\{\sigma_{3}^{*}(N)-N \sigma_{1}^{*}(N)\right\}
\end{aligned}
$$

and

$$
\sum_{k=1}^{N-1} \sigma_{1}^{*}(k) \sigma_{3}^{*}(N-k)=\frac{1}{16}\left(\sigma_{5}^{*}(N)-N \sigma_{3}^{*}(N)\right) .
$$

Recently, Hahn [10] showed that

$$
16 \sum_{k<N} \widetilde{\sigma}_{1}(k) \widetilde{\sigma}_{3}(N-k)=-\widetilde{\sigma}_{5}(N)+2(N-1) \widetilde{\sigma}_{3}(N)+\widetilde{\sigma}_{1}(N) .
$$

It is also interesting to note that the arithmetical functions (for example, $\tau(n)$ and $a(n)$ ), coming out as coefficients of the $q$-series expansion of its corresponding $q$-products, do 
appear in the explicit evaluation of certain convolution sums. For instance, Lahiri (see [11]) proved that

$$
\sum_{k=1}^{N-1} k(N-k) \sigma_{3}(k) \sigma_{3}(N-k)=\frac{1}{540}\left(N^{2} \sigma_{7}(N)-\tau(N)\right)
$$

and from Alaca and Williams (see [12]), we observe that

$$
\begin{aligned}
& \sum_{\substack{(k, m) \in \mathbb{N}^{2} \\
2 k+3 m=N}} \sigma_{1}(k) \sigma_{1}(m) \\
& =\frac{1}{120} \sigma_{3}(N)+\frac{1}{30} \sigma_{3}\left(\frac{N}{2}\right)+\frac{3}{40} \sigma_{3}\left(\frac{N}{3}\right) \\
& \quad+\frac{3}{10} \sigma_{3}\left(\frac{N}{6}\right)+\left(\frac{1}{24}-\frac{N}{12}\right) \sigma_{1}\left(\frac{N}{2}\right)+\left(\frac{1}{24}-\frac{N}{8}\right) \sigma_{1}\left(\frac{N}{3}\right)-\frac{1}{120} a(N) .
\end{aligned}
$$

In [13], Simsek (and also in [14, (2.17)] Simsek along with Ozden and Cagul) has studied other aspects of the arithmetical function $\sigma_{1}(n)$ in connection with the classical Jacobi and Euler functions. We also refer to Kim and Lee [15, Lemma 2.1] and [16].

Thus the study of convolution sums and their applications is classical and they play an important role in number theory. The aim of this article is to first extend and generalize Glaishers formulas (stated in (3) and (4)). We indeed study the sums (in Section 3)

$$
\begin{aligned}
& \sum_{k=1}^{N-1} \sigma_{1}^{*}\left(2^{m} k\right) \sigma_{1}^{*}\left(2^{n}(N-k)\right), \quad \sum_{k=1}^{N-1} \sigma_{1}^{*}\left(2^{m} k\right) \sigma_{3}^{*}\left(2^{n}(N-k)\right), \\
& \sum_{k=1}^{N-1} \sigma_{3}^{*}\left(2^{m} k\right) \sigma_{1}^{*}\left(2^{n}(N-k)\right) \quad \text { and } \sum_{r+s+t=N} \sigma_{1}^{*}\left(2^{m} r\right) \sigma_{1}^{*}\left(2^{n} s\right) \sigma_{1}^{*}\left(2^{l} t\right) .
\end{aligned}
$$

Then, we study and evaluate (in Section 4) sums of the type

$$
\sum_{k=1}^{N-1} \sigma_{1}^{*}\left(3^{m} k ; 3\right) \sigma_{1}^{*}\left(3^{n}(N-k) ; 3\right) \quad \text { and } \sum_{k=1}^{N-1} \sigma_{1}^{*}\left(2^{m} k ; 2\right) \sigma_{1}^{*}\left(3^{n}(N-k) ; 3\right) \text {. }
$$

As applications to our study and evaluations of convolution sums, we show that $\mathcal{A}(q), \mathcal{B}(q)$, and $\mathcal{C}(q)$ are connected by a second-order differential equation (see Theorem 3.8).

In Section 5, we also prove some interesting congruence relations involving the coefficients of modular-like functions and divisor functions (see Theorem 5.3). As a sample, we obtain that if $N \equiv 1(\bmod 8)$, then the congruence

$$
\tau(N) \equiv \sigma_{11}(N) \quad(\bmod 1,415,168)
$$

holds.

In Section 6, we present a generalization of Besge's formula by considering certain combinatorial convolution sums (see Theorem 6.3). It should be noted that Proposition 6.1, Theorem 6.3, and Remark 6.4 exhibit amply the connection between the convolution sums and the Bernoulli numbers. Finally, we record special values of $a(N), \tau(N), b(N), c(N)$, and $l(N)$ (for $1 \leq N \leq 45)$ and some convolution formulas in the Appendix. 


\section{Some weighted convolution sums}

Let $\widehat{f}(a) \widehat{g}(b):=\frac{1}{2}\{f(a) g(b)+f(b) g(a)\}$. It is easily checked that

$$
\sum_{k=1}^{N-1} \widehat{f}(k) \widehat{g}(N-k)=\sum_{k=1}^{N-1} f(k) g(N-k)
$$

Lemma 2.1 For $f, g: \mathbb{N} \rightarrow \mathbb{C}$ and $N \in \mathbb{N}$, we have

$$
\sum_{k=1}^{N-1} \widehat{f}(k) \widehat{g}(N-k)=\frac{N}{2} \sum_{k=1}^{N-1} \widehat{f}(k) \widehat{g}(N-k) .
$$

Proof We observe that

$$
\begin{aligned}
\sum_{k=1}^{N-1} k \widehat{f}(k) \widehat{g}(N-k) & =\frac{1}{2} \sum_{k=1}^{N-1} k\{f(k) g(N-k)+f(N-k) g(k)\} \\
& =\frac{1}{2} \sum_{k=1}^{N-1}(N-k)\{f(N-k) g(k)+f(k) g(N-k)\} \\
& =\sum_{k=1}^{N-1}(N-k) \widehat{f}(k) \widehat{g}(N-k) .
\end{aligned}
$$

Hence

$$
2 \sum_{k=1}^{N-1} \widehat{f}(k) \widehat{g}(N-k)=N \sum_{k=1}^{N-1} \widehat{f}(k) \widehat{g}(N-k)
$$

and thus

$$
\sum_{k=1}^{N-1} \widehat{f}(k) \widehat{g}(N-k)=\frac{N}{2} \sum_{k=1}^{N-1} \widehat{f}(k) \widehat{g}(N-k) .
$$

Lemma 2.2 For $f, g: \mathbb{N} \rightarrow \mathbb{C}$ and $N \in \mathbb{N}$, we have

$$
\sum_{k=1}^{N-1} k^{3} \widehat{f}(k) \widehat{g}(N-k)=-\frac{1}{4} N^{3} \sum_{k=1}^{N-1} \widehat{f}(N-k) \widehat{g}(k)+\frac{3 N}{2} \sum_{k=1}^{N-1} k^{2} \widehat{f}(N-k) \widehat{g}(k) .
$$

Proof We note that

$$
\begin{aligned}
\sum_{k=1}^{N-1} k^{3} \widehat{f}(k) \widehat{g}(N-k)= & \sum_{k=1}^{N-1}(N-k)^{3} \widehat{f}(N-k) \widehat{g}(k) \\
= & N^{3} \sum_{k=1}^{N-1} \widehat{f}(N-k) \widehat{g}(k)-3 N^{2} \sum_{k=1}^{N-1} k \widehat{f}(N-k) \widehat{g}(k) \\
& +3 N \sum_{k=1}^{N-1} k^{2} \widehat{f}(N-k) \widehat{g}(k)-\sum_{k=1}^{N-1} k^{3} \widehat{f}(N-k) \widehat{g}(k) .
\end{aligned}
$$


Then, for the second term on the right-hand side of (6), we replace $k$ by $N-k$ in Lemma 2.1 and obtain

$$
\sum_{k=1}^{N-1}(N-k) \widehat{f}(N-k) \widehat{g}(k)=\frac{N}{2} \sum_{k=1}^{N-1} \widehat{f}(N-k) \widehat{g}(k)
$$

This shows that

$$
\sum_{k=1}^{N-1} \widehat{f}(N-k) \widehat{g}(k)=\frac{N}{2} \sum_{k=1}^{N-1} \widehat{f}(N-k) \widehat{g}(k) .
$$

So, (6) can be written as

$$
\begin{aligned}
& \sum_{k=1}^{N-1} k^{3}\{\widehat{f}(k) \widehat{g}(N-k)+\widehat{f}(N-k) \widehat{g}(k)\} \\
& \quad=-\frac{N^{3}}{2} \sum_{k=1}^{N-1} \widehat{f}(N-k) \widehat{g}(k)+3 N \sum_{k=1}^{N-1} k^{2} \widehat{f}(N-k) \widehat{g}(k) .
\end{aligned}
$$

Here the left-hand side of (7) is

$$
\begin{aligned}
\sum_{k=1}^{N-1} & k^{3}\{\widehat{f}(k) \widehat{g}(N-k)+\widehat{f}(N-k) \widehat{g}(k)\} \\
= & \sum_{k=1}^{N-1} k^{3}\left[\frac{1}{2}\{f(k) g(N-k)+f(N-k) g(k)\}+\frac{1}{2}\{f(N-k) g(k)+f(k) g(N-k)\}\right] \\
= & \sum_{k=1}^{N-1} k^{3}\{f(k) g(N-k)+f(N-k) g(k)\}=2 \sum_{k=1}^{N-1} k^{3} \widehat{f}(k) \widehat{g}(N-k) .
\end{aligned}
$$

Therefore we have

$$
2 \sum_{k=1}^{N-1} k^{3} \widehat{f}(k) \widehat{g}(N-k)=-\frac{1}{2} N^{3} \sum_{k=1}^{N-1} \widehat{f}(N-k) \widehat{g}(k)+3 N \sum_{k=1}^{N-1} k^{2} \widehat{f}(N-k) \widehat{g}(k) .
$$

This completes the proof.

Remark 2.3 In general, we can express

$$
\sum_{k=1}^{N-1} k^{2 l+1} \widehat{f}(k) \widehat{g}(N-k) \quad \text { for } k \in \mathbb{N} \cup\{0\}
$$

as a combination in terms of the sum

$$
\sum_{k=1}^{N-1} k^{2} \widehat{f}(k) \widehat{g}(N-k) \quad \text { with } j=0,1,2, \ldots, k
$$

with their coefficients being polynomials in $N$ of degree at most $(2 l+1)$. 
Lemma 2.4 For $l, N \in \mathbb{N}$ and $f, g: \mathbb{N} \rightarrow \mathbb{C}$, we have

$$
\left\{1+(-1)^{l+1}\right\} \sum_{k=1}^{N-1} k \widehat{f}(k) \widehat{g}(N-k)=\sum_{k=1}^{N-1} \widehat{f}(N-k) \widehat{g}(k)\left\{\sum_{j=0}^{l-1}\left(\begin{array}{l}
l \\
j
\end{array}\right)(-1)^{j} k^{j} N^{l-j}\right\} .
$$

Proof We note that (for $l \in \mathbb{N})$,

$$
\begin{aligned}
& \sum_{k=1}^{N-1} k \widehat{f}(k) \widehat{g}(N-k)=\sum_{k=1}^{N-1}(N-k) \widehat{f}(N-k) \widehat{g}(k) \\
& =\sum_{k=1}^{N-1} \widehat{f}(N-k) \widehat{g}(k)\left\{\sum_{j=0}^{l}\left(\begin{array}{l}
l \\
j
\end{array}\right)(-1)^{j} k^{j} N^{l-j}\right\} \\
& =(-1)^{l} \sum_{k=1}^{N-1} k \widehat{f}(N-k) \widehat{g}(k)+\sum_{k=1}^{N-1} \widehat{f}(N-k) \widehat{g}(k)\left\{\sum_{j=0}^{l-1}\left(\begin{array}{l}
l \\
j
\end{array}\right)(-1)^{j} k^{j} N^{l-j}\right\} .
\end{aligned}
$$

Observing the fact that

$$
\sum_{k=1}^{N-1} k \widehat{f}(k) \widehat{g}(N-k)=\sum_{k=1}^{N-1} k^{\widehat{f}}(N-k) \widehat{g}(k)
$$

for any $l \in \mathbb{N}$, we obtain

$$
\left\{1+(-1)^{l+1}\right\} \sum_{k=1}^{N-1} k^{l} \widehat{f}(k) \widehat{g}(N-k)=\sum_{k=1}^{N-1} \widehat{f}(N-k) \widehat{g}(k)\left\{\sum_{j=0}^{l-1}\left(\begin{array}{l}
l \\
j
\end{array}\right)(-1)^{j} k^{j} N^{l-j}\right\} .
$$

This proves Lemma 2.4 .

Remark 2.5 By iteration process, in principle, the convolution sum $\sum_{k=1}^{N-1} k \widehat{f}(k) \widehat{g}(N-k)$ can be evaluated for any odd $l \in \mathbb{N}$.

Let

$$
\begin{aligned}
\widehat{f}(a) \widehat{g}(b) \widehat{h}(c):= & \frac{1}{6}\{f(a) g(b) h(c)+f(a) g(c) h(b)+f(b) g(a) h(c) \\
& +f(b) g(c) h(a)+f(c) g(a) h(b)+f(c) g(b) h(a)\} .
\end{aligned}
$$

Lemma 2.6 For $f: \mathbb{N} \rightarrow \mathbb{C}$ and $N \in \mathbb{N}$. Then we have

$$
\sum_{\substack{(r, s, t) \in \mathbb{N}^{3} \\
r+s+t=N}} \widehat{f}(r) \widehat{f}(s) \widehat{f}(t)=\frac{N}{3} \sum_{\begin{array}{c}
(r, s, t) \in \mathbb{N}^{3} \\
r+s+t=N
\end{array}} \widehat{f}(r) \widehat{f}(s) \widehat{f}(t)
$$

Proof From the cyclic transformation, $r \rightarrow s \rightarrow t \rightarrow r$, we observe that

$$
\sum_{\substack{(r, s, t) \in \mathbb{N}^{3} \\ r+s+t=N}} r \widehat{f}(r) \widehat{f}(s) \widehat{f}(t)=\sum_{\substack{(r, s, t) \in \mathbb{N}^{3} \\ r+s+t=N}} \widehat{f}(r) \widehat{f}(s) \widehat{f}(t)=\sum_{\substack{(r, s, t) \in \mathbb{N}^{3} \\ r+s+t=N}} \widehat{t f}(r) \widehat{f}(s) \widehat{f}(t) .
$$


Therefore, we have

$$
\sum_{\substack{(r, s, t) \in \mathbb{N}^{3} \\ r+s+t=N}} N \widehat{f}(r) \widehat{f}(s) \widehat{f}(t)=\sum_{\substack{(r, s, t) \in \mathbb{N}^{3} \\ r+s+t=N}}(r+s+t) \widehat{f}(r) \widehat{f}(s) \widehat{f}(t)=3 \sum_{\substack{(r, s, t) \in \mathbb{N}^{3} \\ r+s+t=N}} r \widehat{f}(r) \widehat{f}(s) \widehat{f}(t) .
$$

Now Lemma 2.6 follows.

Lemma 2.7 For $f: \mathbb{N} \rightarrow \mathbb{C}$ and $N \in \mathbb{N}$. Then we have (with $g(m)=m f(m)$ for all $m \in \mathbb{N})$

$$
3 \sum_{\substack{(r, s, t) \in \mathbb{N}^{3} \\ r+s+t=N}} r^{2} f(r) f(s) f(t)=N^{2} \sum_{\substack{(r, s, t) \in \mathbb{N}^{3} \\ r+s+t=N}} f(r) f(s) f(t)+6 \sum_{t=1}^{N-2} \sum_{\substack{(r, s) \in \mathbb{N}^{2} \\ r+s=N-t}} g(r) g(s) .
$$

Proof As in Lemma 2.6, again by the cyclic transformation $r \rightarrow s \rightarrow t \rightarrow r$, we observe that

$$
\sum_{\substack{(r, s, t) \in \mathbb{N}^{3} \\ r+s+t=N}} r^{2} f(r) f(s) f(t)=\sum_{\substack{(r, s, t) \in \mathbb{N}^{3} \\ r+s+t=N}} s^{2} f(r) f(s) f(t)=\sum_{\substack{(r, s, t) \in \mathbb{N}^{3} \\ r+s+t=N}} t^{2} f(r) f(s) f(t)
$$

and

$$
\sum_{\substack{(r, s, t) \in \mathbb{N}^{3} \\ r+s+t=N}} r s f(r) f(s) f(t)=\sum_{\substack{(r, s, t) \in \mathbb{N}^{3} \\ r+s+t=N}} s t f(r) f(s) f(t)=\sum_{\substack{(r, s, t) \in \mathbb{N}^{3} \\ r+s+t=N}} \operatorname{trf}(r) f(s) f(t) .
$$

Therefore, we get (from (8) and (9))

$$
\begin{aligned}
\sum_{\substack{(r, s, t) \in \mathbb{N}^{3} \\
r+s+t=N}} n^{2} f(r) f(s) f(t) & =3 \sum_{\substack{(r, s, t) \in \mathbb{N}^{3} \\
r+s+t=N}} r^{2} f(r) f(s) f(t)+6 \sum_{\substack{(r, s, t) \in \mathbb{N}^{3} \\
r+s+t=N}} r s f(r) f(s) f(t) \\
& =3 \sum_{\substack{(r, s, t) \in \mathbb{N}^{3} \\
r+s+t=N}} r^{2} f(r) f(s) f(t)+6 \sum_{t=1}^{N-2} f(t) \sum_{\substack{(r, s) \in \mathbb{N}^{2} \\
r+s=N-t}} g(r) g(s),
\end{aligned}
$$

where $g(m)=m f(m): \mathbb{N} \rightarrow \mathbb{C}$ for all $m \in \mathbb{N}$. This proves Lemma 2.7.

\section{The convolution sum $\sum_{k=1}^{N-1} \sigma_{1}^{*}(k) \sigma_{1}^{*}(N-k)$ and its extensions}

Proposition 3.1 (a) [17, Theorem 15.1, p.184], we have

$$
\begin{aligned}
\sum_{k<N / 2} \sigma(k) \sigma(N-2 k)= & \frac{1}{24}\left\{2 \sigma_{3}(N)+(1-3 N) \sigma(N)+8 \sigma_{3}(N / 2)\right. \\
& +(1-6 N) \sigma(N / 2)\} .
\end{aligned}
$$

(b) [3, Theorem 3], [17, Theorem 15.3, p.188], we have

$$
\begin{aligned}
& \sum_{k<N / 3} \sigma_{1}(k) \sigma_{1}(N-3 k) \\
& \quad=\frac{1}{24}\left\{\sigma_{3}(N)+(1-2 N) \sigma_{1}(N)+9 \sigma_{3}\left(\frac{N}{3}\right)+(1-6 N) \sigma_{1}\left(\frac{N}{3}\right)\right\} .
\end{aligned}
$$


Proposition 3.2 Let $s, N \in \mathbb{N}$. Then we obtain

$$
\sigma_{s}^{*}(N ; p)=\sigma_{s}(N)-\sigma_{s}(N / p) .
$$

In particular, we have

$$
\sigma_{s}^{*}(N)=\sigma_{s}(N)-\sigma_{s}(N / 2)
$$

which can also be seen in [17, p.27].

Proof We can know that

$$
\sigma_{s}^{*}(N ; p):=\sum_{\substack{d \mid N \\ \frac{N}{d} \neq 0(\bmod p)}} d^{s}=\sum_{d \mid N} d^{s}-\sum_{\substack{d \mid N \\ \frac{N}{d} \equiv 0(\bmod p)}} d^{s}=\sigma_{s}(N)-\sum_{d \mid \frac{N}{p}} d^{s}=\sigma_{s}(N)-\sigma_{s}(N / p) .
$$

Proposition 3.3 Let $N$ be any positive integer. For $m, n \in \mathbb{N} \cup\{0\}$, where $0 \leq m \leq n$, we have
(a) $\sum_{k=1}^{N-1} \sigma_{1}^{*}\left(2^{m} k\right) \sigma_{1}^{*}\left(2^{n}(N-k)\right)=2^{n+m-2}\left(\sigma_{3}^{*}(N)-N \sigma_{1}^{*}(N)\right)$.
(b) $\sum_{k=1}^{N-1} \sigma_{1}^{*}\left(2^{m} k\right) \sigma_{3}^{*}\left(2^{n}(N-k)\right)=2^{3 n+m-4}\left(\sigma_{5}^{*}(N)-N \sigma_{3}^{*}(N)\right)$.
(c) $\sum_{k=1}^{N-1} \sigma_{3}^{*}\left(2^{m} k\right) \sigma_{1}^{*}\left(2^{n}(N-k)\right)=2^{n+3 m-4}\left(\sigma_{5}^{*}(N)-N \sigma_{3}^{*}(N)\right)$.
(d) $\sum_{k=1}^{N-1} \sigma_{1}^{*}\left(2^{m} k\right)\left\{\sigma_{3}^{*}\left(2^{n+1}(N-k)\right)-k \sigma_{1}^{*}\left(2^{3 n+2}(N-k)\right)\right\}$

$$
=2^{3 n+m-1}\left(\sigma_{5}^{*}(N)-N^{2} \sigma_{1}^{*}(N)\right) .
$$

Proof For the sake of completeness, we just hint the proof of (b). We note that $\sigma_{k}^{*}\left(2^{a}\right)=2^{a k}$. Since $\sigma_{k}^{*}$ is a $2^{k}$-scalar multiplicative function (i.e., $\left.\sigma_{k}^{*}(2 N)=\sigma_{k}^{*}(2) \cdot \sigma_{k}^{*}(N)\right)$, we have

$$
\sum_{k=1}^{N-1} \sigma_{1}^{*}\left(2^{m} k\right) \sigma_{3}^{*}\left(2^{n}(N-k)\right)=2^{m+3 n} \sum_{k=1}^{N-1} \sigma_{1}^{*}(k) \sigma_{3}^{*}(N-k)=2^{3 n+m-4}\left(\sigma_{5}^{*}(N)-N \sigma_{3}^{*}(N)\right)
$$

by (4). The proofs of (a), (c), and (d) are similar to (b).

Remark 3.4 Using Eq. (3) and Lemma 2.1, we obtain

$$
\sum_{k=1}^{N-1} k \sigma_{1}^{*}(k) \sigma_{1}^{*}(N-k)=\frac{N}{2} \sum_{k=1}^{N-1} \sigma_{1}^{*}(k) \sigma_{1}^{*}(N-k)=\frac{N}{8}\left\{\sigma_{3}^{*}(N)-N \sigma_{1}^{*}(N)\right\} .
$$

Theorem 3.5 Let $N(\geq 3)$ be any integer with $m, n, l \in \mathbb{N} \cup\{0\}$. Then we have

$$
\sum_{r+s+t=N} \sigma_{1}^{*}\left(2^{m} r\right) \sigma_{1}^{*}\left(2^{n} s\right) \sigma_{1}^{*}\left(2^{l} t\right)=\frac{2^{m+n+l}}{64}\left(\sigma_{5}^{*}(N)-3 N \sigma_{3}^{*}(N)+2 N^{2} \sigma_{1}^{*}(N)\right) .
$$


Proof Let $r_{1} l_{1}=p^{m_{1}} N$ and $l_{1} \not \equiv 0(\bmod p)$ for some $r_{1}, l_{1} \in \mathbb{N}$ and prime $p$. Since $p$ does not divide $l_{1}$, we can write $r_{1}=p^{m_{1}} d$ for some $d \in \mathbb{N}$. Therefore, we have

$$
\begin{aligned}
\sigma_{s}^{*}\left(p^{m_{1}} N ; p\right) & =\sum_{\substack{r_{1} \mid p^{m_{1}} \\
\frac{p^{m_{1}}}{r_{1}} \neq 0(\bmod p)}} r_{1}^{s}=\sum_{\substack{d \mid N \\
\frac{N}{d} \neq 0(\bmod p)}}\left(p^{m_{1}} d\right)^{s} \\
& =p^{m_{1} s} \sum_{\substack{d \mid N \\
\frac{N}{d} \neq 0(\bmod p)}} d^{s}=p^{m_{1} s} \sigma_{s}^{*}(N ; p) .
\end{aligned}
$$

We note that

$$
\begin{aligned}
& \sum_{r+s+t=N} \sigma_{1}^{*}\left(2^{m} r\right) \sigma_{1}^{*}\left(2^{n} s\right) \sigma_{1}^{*}\left(2^{l} t\right) \\
& =2^{m+n+l} \sum_{t=1}^{N-2} \sigma_{1}^{*}(t) \sum_{r+s=N-t} \sigma_{1}^{*}(r) \sigma_{1}^{*}(s) \\
& =2^{m+n+l} \sum_{t=1}^{N-2} \sigma_{1}^{*}(t)\left\{\frac{1}{4}\left(\sigma_{3}^{*}(N-t)-(N-t) \sigma_{1}^{*}(N-t)\right)\right\} \quad(\text { by }(3)) \\
& =\frac{2^{m+n+l}}{4}\left\{\sum_{t=1}^{N-2} \sigma_{1}^{*}(t) \sigma_{3}^{*}(N-t)-\sum_{t=1}^{N-2}(N-t) \sigma_{1}^{*}(t) \sigma_{1}^{*}(N-t)\right\} \\
& =\frac{2^{m+n+l}}{4}\left\{\sum_{t=1}^{N-2} \sigma_{1}^{*}(t) \sigma_{3}^{*}(N-t)-\sum_{k=1}^{N-2} k \sigma_{1}^{*}(N-k) \sigma_{1}^{*}(k)\right\}
\end{aligned}
$$

and hence, we use Eq. (1) and Eq. (10) to obtain the result.

\section{Corollary 3.6 Let}

$$
\begin{aligned}
f(p) \in & \left\{\sum_{k=1}^{p-1} \sigma_{1}^{*}\left(2^{m} k\right) \sigma_{1}^{*}\left(2^{n}(p-k)\right), \sum_{k=1}^{p-1} \sigma_{1}^{*}\left(2^{m} k\right) \sigma_{3}^{*}\left(2^{n}(p-k)\right),\right. \\
& \left.\sum_{r+s+t=p} \sigma_{1}^{*}\left(2^{m} r\right) \sigma_{1}^{*}\left(2^{n} s\right) \sigma_{1}^{*}\left(2^{l} t\right)\right\},
\end{aligned}
$$

where $p=2 q+1$ is an odd prime. Then

$$
f(p)=a S_{4}(q+1)+b S_{3}(q+1)+c S_{2}(q+1)+d S_{1}(q+1),
$$

where the coefficients $a, b, c, d$ are listed in Table 1 .

Table 1 Coefficients for $p=2 q+1$

\begin{tabular}{lllll}
\hline $\boldsymbol{f}(\boldsymbol{p}):=$ & $\boldsymbol{a}$ & $\boldsymbol{b}$ & $\boldsymbol{c}$ & $\boldsymbol{d}$ \\
\hline$\sum_{k=1}^{p-1} \sigma_{1}^{*}\left(2^{m} k\right) \boldsymbol{\sigma}_{1}^{*}\left(2^{n}(p-k)\right)$ & 0 & 0 & $3 \cdot 2^{m+n+1}$ & $-2^{m+n+1}$ \\
$\sum_{k=1}^{p-1} \sigma_{1}^{*}\left(2^{m} k\right) \sigma_{3}^{*}\left(2^{n}(p-k)\right)$ & $5 \cdot 2^{m+3 n+1}$ & $-2^{m+3 n+2}$ & $5 \cdot 2^{m+3 n}$ & $-2^{m+3 n}$ \\
$\sum_{r+s+t=p} \sigma_{1}^{*}\left(2^{m} r\right) \sigma_{1}^{*}\left(2^{n} s\right) \sigma_{1}^{*}\left(2^{l} t\right)$ & $5 \cdot 2^{m+n+l-1}$ & $-3 \cdot 2^{m+n+1}$ & $2^{m+n+1+1}$ & $-2^{m+n+1-1}$ \\
\hline
\end{tabular}


Table 2 Convolution formulas for prime $p=2 q+1$

\begin{tabular}{ll}
\hline Convolution sum & $\boldsymbol{f}(\mathbf{2 q}+\mathbf{1}):=$ \\
\hline$\sum_{k=1}^{p-1} \sigma_{1}(k) \sigma_{1}(p-k)$ & $\frac{1}{3} q\left(10 q^{2}+9 q-1\right)$ \\
$\sum_{k=1}^{p-1} \sigma_{1}^{*}(k) \sigma_{1}^{*}(p-k)$ & $2 q^{2}(q+1)$ \\
$\sum_{k=1}^{p-1} \sigma_{1}(k) \sigma_{3}(p-k)$ & $\frac{1}{15} q\left(42 q^{4}+75 q^{3}+50 q^{2}+15 q-2\right)$ \\
$\sum_{k=1}^{p-1} \sigma_{1}^{*}(k) \sigma_{3}^{*}(p-k)$ & $q^{2}(q+1)\left(2 q^{2}+2 q+1\right)$ \\
$\sum_{r+s+t=p} \sigma_{1}(r) \sigma_{1}(s) \sigma_{1}(t)$ & $\frac{1}{12} q^{2}\left(14 q^{3}+5 q^{2}-8 q+1\right)$ \\
$\sum_{r+s+t=p} \sigma_{1}^{*}(r) \sigma_{1}^{*}(s) \sigma_{1}^{*}(t)$ & $\frac{1}{2} q^{4}(q+1)$ \\
\hline
\end{tabular}

Table 3 Convolution formulas

\begin{tabular}{ll}
\hline Convolution sum & \\
\hline$A(N):=\sum_{k=1}^{N-1} \sigma_{1}(k) \sigma_{1}(N-k)$ & $\frac{1}{12}\left(5 \sigma_{3}(N)+(1-6 N) \sigma_{1}(N)\right)$ \\
$B(N):=\sum_{k=1}^{N-1} \sigma_{1}^{*}(k) \sigma_{1}^{*}(N-k)$ & $\frac{1}{4}\left(\sigma_{3}^{*}(N)-N \sigma_{1}^{*}(N)\right)$ \\
$C(N):=\sum_{r+s+t=N} \sigma_{1}(r) \sigma_{1}(s) \sigma_{1}(t)$ & $\frac{7}{192} \sigma_{5}(N)+\left(\frac{5}{96}-\frac{5}{32} N\right) \sigma_{3}(N)$ \\
& $+\left(\frac{1}{192}-\frac{1}{16} N+\frac{1}{8} N^{2}\right) \sigma_{1}(N)$ \\
$D(N):=\sum_{r+s+t=N} \sigma_{1}^{*}(r) \sigma_{1}^{*}(s) \sigma_{1}^{*}(t)$ & $\frac{1}{64}\left(\sigma_{5}^{*}(N)-3 N \sigma_{3}^{*}(N)+2 N^{2} \sigma_{1}^{*}(N)\right)$ \\
\hline
\end{tabular}

Remark 3.7 If $p=2 q+1$ is any odd prime, then

$$
\begin{aligned}
\sum_{k=1}^{2 q} \sigma_{1}^{*}(k) \sigma_{1}^{*}(2 q+1-k) & \equiv \sum_{k=1}^{2 q} \sigma_{1}^{*}(k) \sigma_{3}^{*}(2 q+1-k) \\
& \equiv \sum_{r+s+t=2 q+1} \sigma_{1}^{*}(r) \sigma_{1}^{*}(s) \sigma_{1}^{*}(t) \\
& \equiv 0 \quad\left(\bmod S_{1}(q+1)\right)
\end{aligned}
$$

from Table 2.

Now, we compare the values of the convolution sums in Table 3 [17, p.148]. For almost all $N \in \mathbb{N}$, we find that $A(N)>B(N)$ and $C(N)>D(N)$ (Figure 1). As an application to Theorem 3.5, we have the following.

Theorem 3.8 The q-series $\mathcal{A}(q), \mathcal{B}(q), \mathcal{C}(q)$ are connected by the differential equation,

$$
64 \mathcal{A}^{3}(q)=\mathcal{C}(q)-3 q \frac{d \mathcal{B}(q)}{d q}+2 q^{2} \frac{d^{2} \mathcal{A}(q)}{d q^{2}}+2 q \frac{d \mathcal{A}(q)}{d q}
$$

where $q \frac{d \mathcal{B}(q)}{d q}=\sum_{N=1}^{\infty} N \sigma_{3}^{*}(N) q^{N}$ and $q^{2} \frac{d^{2} \mathcal{A}(q)}{d q^{2}}=\sum_{N=1}^{\infty} N(N-1) \sigma_{1}^{*}(N) q^{N}$.

Proof We note that

$$
\begin{aligned}
64 \mathcal{A}^{3}(q) & =64\left(\sum_{l=1}^{\infty} \sigma_{1}^{*}(l) q^{l}\right)\left(\sum_{m=1}^{\infty} \sigma_{1}^{*}(m) q^{m}\right)\left(\sum_{n=1}^{\infty} \sigma_{1}^{*}(n) q^{n}\right) \\
& =\sum_{N=3}^{\infty}\left(64 \sum_{l+m+n=N} \sigma_{1}^{*}(l) \sigma_{1}^{*}(m) \sigma_{1}^{*}(n)\right) q^{N} \\
& =\sum_{N=3}^{\infty}\left\{\sigma_{5}^{*}(N)-3 N \sigma_{3}^{*}(N)+2 N^{2} \sigma_{1}^{*}(N)\right\} q^{N}
\end{aligned}
$$



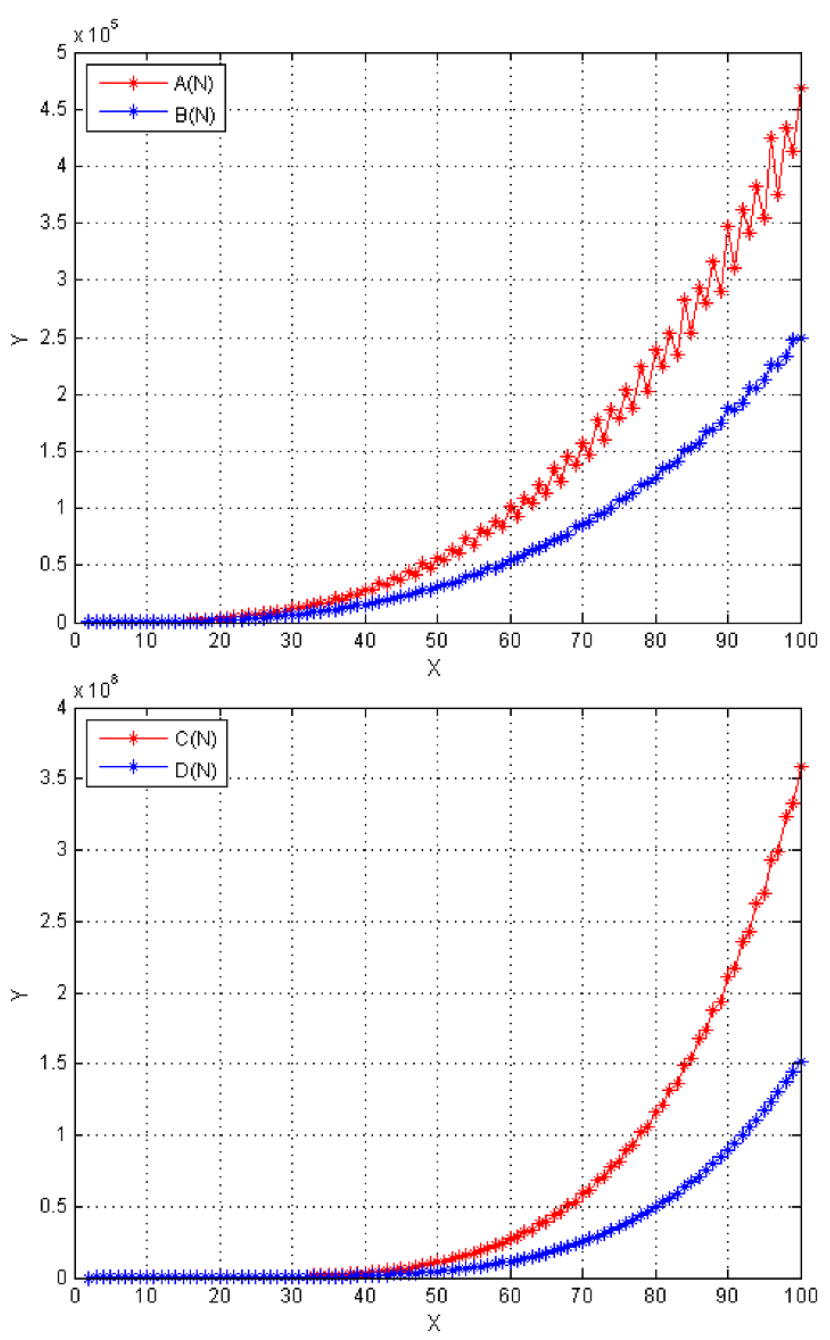

Figure $1 A(N), B(N), C(N)$, and $D(N)$.

from Theorem 3.5. We note that $\sigma_{5}^{*}(N)-3 N \sigma_{3}^{*}(N)+2 N^{2} \sigma_{1}^{*}(N)$ is zero for $N=1$ and $N=2$. Thus the differential Eq. (12) follows.

Remark 3.9 We also note that from Eq. (1) and Eq. (3), we can determine the equations

$$
16 \mathcal{A}(q) \mathcal{B}(q)=\mathcal{C}(q)-q \frac{d \mathcal{B}(q)}{d q}
$$

and

$$
4 \mathcal{A}^{2}(q)=\mathcal{B}(q)-q \frac{d \mathcal{A}(q)}{d q}
$$

From (14) and (15), Eq. (12) can also be deduced. Using [18, (28)] and (15), we also get

$$
\wp^{\prime \prime}\left(\frac{\tau}{2}, \tau\right)=32 \pi^{4}\left(4 \mathcal{A}^{2}(q)+q \frac{d \mathcal{A}(q)}{d q}\right)
$$


where

$$
\wp\left(z ; \Lambda_{\tau}\right)=\frac{1}{z^{2}}+\sum_{\substack{\omega \in \Lambda_{\tau}, \omega \neq 0}}\left\{\frac{1}{(z-\omega)^{2}}-\frac{1}{\omega^{2}}\right\}
$$

and $\Lambda_{\tau}=\mathbb{Z}+\tau \mathbb{Z}(\tau \in \mathcal{H}$ the complex upper-half plane) is a lattice and $z \in \mathbb{C}$.

\section{The convolution sum $\sum_{k=1}^{N-1} \sigma_{1}^{*}(k ; 3) \sigma_{1}^{*}(N-k ; 3)$ and its extensions}

Theorem 4.1 Let $N$ be a positive integer. And let $m, n \in \mathbb{N} \cup\{0\}$. Then we have

$$
\sum_{k=1}^{N-1} \sigma_{1}^{*}\left(3^{m} k ; 3\right) \sigma_{1}^{*}\left(3^{n}(N-k) ; 3\right)=3^{n+m-1}\left\{\sigma_{3}^{*}(N ; 3)-N \sigma_{1}^{*}(N ; 3)\right\} .
$$

Proof From Proposition 3.2 and Eq. (11), we can deduce that

$$
\begin{aligned}
\sum_{k=1}^{N-1} \sigma_{1}^{*}\left(3^{m} k ; 3\right) \sigma_{1}^{*}\left(3^{n}(N-k) ; 3\right) & \\
= & 3^{n+m} \sum_{k=1}^{N-1}\left\{\sigma_{1}(k)-\sigma_{1}\left(\frac{k}{3}\right)\right\}\left\{\sigma_{1}(N-k)-\sigma_{1}\left(\frac{N-k}{3}\right)\right\} \\
= & 3^{n+m}\left\{\sum_{k=1}^{N-1} \sigma_{1}(k) \sigma_{1}(N-k)-\sum_{k=1}^{N-1} \sigma_{1}(k) \sigma_{1}\left(\frac{N-k}{3}\right)\right. \\
& \left.-\sum_{k=1}^{N-1} \sigma_{1}\left(\frac{k}{3}\right) \sigma_{1}(N-k)+\sum_{k=1}^{N-1} \sigma_{1}\left(\frac{k}{3}\right) \sigma_{1}\left(\frac{N-k}{3}\right)\right\} .
\end{aligned}
$$

Now, we note that

$$
\begin{aligned}
& \sum_{k=1}^{N-1} \sigma_{1}(k) \sigma_{1}\left(\frac{N-k}{3}\right)=\sum_{t<N / 3} \sigma_{1}(N-3 t) \sigma_{1}(t) \\
& \sum_{k=1}^{N-1} \sigma_{1}\left(\frac{k}{3}\right) \sigma_{1}(N-k)=\sum_{t<N / 3} \sigma_{1}(t) \sigma_{1}(N-3 t) \\
& \sum_{k=1}^{N-1} \sigma_{1}\left(\frac{k}{3}\right) \sigma_{1}\left(\frac{N-k}{3}\right)=\sum_{t<N / 3} \sigma_{1}(t) \sigma_{1}\left(\frac{N}{3}-t\right) .
\end{aligned}
$$

Therefore, the theorem follows from Eq. (1) and Proposition 3.1(b).

Theorem 4.2 Let $N$ be a positive integer and let $m, n \in \mathbb{N} \cup\{0\}$. Then we have

$$
\begin{aligned}
& \sum_{k=1}^{N-1} \sigma_{1}^{*}\left(2^{m} k ; 2\right) \sigma_{1}^{*}\left(3^{n}(N-k) ; 3\right) \\
& \quad=\frac{2^{m} \cdot 3^{n}}{120}\left[36\left\{\sigma_{3}^{*}(N)-\sigma_{3}^{*}\left(\frac{N}{3}\right)\right\}-20 N \sigma_{1}^{*}(N)-15 N \sigma_{1}^{*}(N ; 3)-a(N)\right] .
\end{aligned}
$$


Table $4 \sum_{k=1}^{N-1} \sigma_{1}^{*}\left(2^{m} k ; 2\right) \sigma_{1}^{*}\left(3^{n}(N-k) ; 3\right)$ and $\sum_{k=1}^{N-1} \sigma_{1}\left(2^{m} k\right) \sigma_{1}\left(3^{n}(N-k)\right)$

\begin{tabular}{cc}
\hline & Convolution formula \\
\hline$\sum_{k=1}^{N-1} \sigma_{1}^{*}\left(2^{m} k ; 2\right) \sigma_{1}^{*}\left(3^{n}(N-k) ; 3\right)$ & $\frac{2^{m} \cdot 3^{n}}{120}\left[36\left\{\sigma_{3}^{*}(N)-\sigma_{3}^{*}\left(\frac{N}{3}\right)\right\}-20 N \sigma_{1}^{*}(N)\right.$ \\
& $\left.-15 N \sigma_{1}^{*}(N ; 3)-a(N)\right]$ \\
$\sum_{k=1}^{N-1} \sigma_{1}\left(2^{m} k\right) \sigma_{1}\left(3^{n}(N-k)\right)$ & $\frac{1}{240}\left[\left(2^{m+3}-3\right)\left(3^{n+3}-7\right) \sigma_{3}(N)\right.$ \\
& $-8\left(2^{m}-1\right)\left(3^{n+3}-7\right) \sigma_{3}\left(\frac{N}{2}\right)$ \\
& $-27\left(2^{m+3}-3\right)\left(3^{n}-1\right) \sigma_{3}\left(\frac{N}{3}\right)$ \\
& $+216\left(2^{m}-1\right)\left(3^{n}-1\right) \sigma_{3}\left(\frac{N}{6}\right)$ \\
& $-5\left\{3-2^{m+2}-3^{n+1}\right.$ \\
& $\left.+6\left(7 \cdot 2^{m} \cdot 3^{n}-2 \cdot 3^{n}-2^{m}\right) N\right\} \sigma_{1}(N)$ \\
& $+20\left(2^{m}-1\right)\left(2 \cdot 3^{n+1} N-1\right) \sigma_{1}\left(\frac{N}{2}\right)$ \\
& $+15\left(3^{n}-1\right)\left(3 \cdot 2^{m+1} N-1\right) \sigma_{1}\left(\frac{N}{3}\right)$ \\
& $\left.-6\left(2^{m}-1\right)\left(3^{n}-1\right) a(N)\right]$ \\
\hline
\end{tabular}

Table 5 Some convolution formulas

\begin{tabular}{ll}
\hline Convolution sum & Convolution formula \\
\hline$\sum_{k=1}^{N-1} \sigma_{1}^{*}(k) \sigma_{5}^{*}(N-k)$ & $\frac{1}{408}\left\{12 \sigma_{7}^{*}(N)-17 N \sigma_{5}^{*}(N)+5 b(N)\right\}$ \\
$\sum_{k=1}^{N-1} \sigma_{3}^{*}(k) \sigma_{3}^{*}(N-k)$ & $\frac{1}{136}\left\{\sigma_{7}^{*}(N)-b(N)\right\}$ \\
$\sum_{k=1}^{N-1} \sigma_{1}^{*}(k) \sigma_{7}^{*}(N-k)$ & $\frac{1}{992}\left\{17 \sigma_{9}^{*}(N)-31 N \sigma_{7}^{*}(N)+448 /(N)+14 c(N)\right\}$ \\
$\sum_{k=1}^{N-1} \sigma_{3}^{*}(k) \sigma_{5}^{*}(N-k)$ & $\frac{1}{496}\left\{\sigma_{9}^{*}(N)-32 /(N)-c(N)\right\}$ \\
$\sum_{k=1}^{N-1} \sigma_{5}^{*}(k) \sigma_{5}^{*}(N-k)$ & $\frac{1}{2,764}\left\{\sigma_{11}^{*}(N)-\tau(N)+692 \tau\left(\frac{N}{2}\right)\right\}$ \\
$\sum_{k=1}^{N-1} \sigma_{1}^{*}(k) \sigma_{9}^{*}(N-k)$ & $\frac{1}{27,640}\left\{310 \sigma_{11}^{*}(N)-691 N \sigma_{9}^{*}(N)+381 \tau(N)+109,488 \tau\left(\frac{N}{2}\right)\right\}$ \\
$\sum_{k=1}^{N-1} \sigma_{3}^{*}(k) \sigma_{7}^{*}(N-k)$ & $\frac{1}{22,112}\left\{17 \sigma_{11}^{*}(N)-17 \tau(N)-13,112 \tau\left(\frac{N}{2}\right)\right\}$ \\
\hline
\end{tabular}

Proof We note that

$$
\begin{aligned}
& \sum_{k=1}^{N-1} \sigma_{1}^{*}\left(2^{m} k ; 2\right) \sigma_{1}^{*}\left(3^{n}(N-k) ; 3\right) \\
& =2^{m} \cdot 3^{n} \sum_{k=1}^{N-1}\left\{\sigma_{1}(k)-\sigma_{1}\left(\frac{k}{2}\right)\right\}\left\{\sigma_{1}(N-k)-\sigma_{1}\left(\frac{N-k}{3}\right)\right\} \\
& =2^{m} \cdot 3^{n}\left\{\sum_{k=1}^{N-1} \sigma_{1}(k) \sigma_{1}(N-k)-\sum_{k<N / 3} \sigma_{1}(N-3 k) \sigma_{1}(k)\right. \\
& \left.\quad-\sum_{k<N / 2} \sigma_{1}(k) \sigma_{1}(N-2 k)+\sum_{2 k+3 m=N} \sigma_{1}(k) \sigma_{1}(m)\right\} .
\end{aligned}
$$

Now, the theorem follows from (1), Proposition 3.1(a), (b), and Eq. (5).

Remark 4.3 We can compare the two sums

$$
\sum_{k=1}^{N-1} \sigma_{1}^{*}\left(2^{m} k ; 2\right) \sigma_{1}^{*}\left(3^{n}(N-k) ; 3\right), \quad \sum_{k=1}^{N-1} \sigma_{1}\left(2^{m} k\right) \sigma_{1}\left(3^{n}(N-k)\right)
$$

as follows (see Table 4). Here we find the formula for the sum $\sum_{k=1}^{N-1} \sigma_{1}\left(2^{m} k\right) \sigma_{1}\left(3^{n}(N-k)\right)$ in a similar way as in Theorem 4.2 (see [16, Theorem 2.5]).

\section{Congruence relations of coefficients of certain modular-like functions}

Lemma 5.1 Let $n \geq 2$. Then we have Table 5 . 
Proof We use Table 15 in the Appendix, and the proof of Lemma 5.1 is now similar to the proof of Theorem 4.1.

Remark 5.2 It is easy to observe that

$$
\sum_{k=1}^{N-1} \sigma_{5}^{*}(k) \sigma_{5}^{*}(N-k)=\frac{8}{17} \sum_{k=1}^{N-1} \sigma_{3}^{*}(k) \sigma_{7}^{*}(N-k)
$$

when $N$ is odd.

As an application to the explicit evaluation of convolution sums, using Table 5 we prove the following theorem.

Theorem 5.3 If $N$ is odd, then

(a) $b(N) \equiv N \sigma_{5}(N)(\bmod 48)$. In particular $N \equiv 1(\bmod 8)$, we have $b(N) \equiv N \sigma_{5}(N)(\bmod 192)$.

(b) $b(N) \equiv \sigma_{7}(N)(\bmod 2,176)$. In particular $N \equiv 1(\bmod 8)$, we have $b(N) \equiv \sigma_{7}(N)(\bmod 17,408)$.

(c) $\tau(N) \equiv \sigma_{11}(N)(\bmod 176,896)$. In particular $N \equiv 1(\bmod 8)$, we have $\tau(N) \equiv \sigma_{11}(N)(\bmod 1,415,168)$.

Proof Since the proofs of (a), (b), and (c) are similar, we only prove (c). When $N$ is odd, from Table 5 , we see that

$$
5,528 \sum_{k=1}^{\frac{N-1}{2}} \sigma_{5}^{*}(k) \sigma_{5}^{*}(N-k)=\sigma_{11}(N)-\tau(N) .
$$

Since $N$ is odd, $k \not \equiv N-k(\bmod 2)$, and hence either $k$ is even or $N-k$ is even. Then, by (11), $\sigma_{5}^{*}(k)=2^{5} \sigma_{5}^{*}\left(\frac{k}{2}\right)$ or $\sigma_{5}^{*}(N-k)=2^{5} \sigma_{5}^{*}\left(\frac{N-k}{2}\right)$. So, in general,

$$
\tau(N) \equiv \sigma_{11}(N) \quad(\bmod 176,896)
$$

In particular, for $N \equiv 1(\bmod 8)$, we have Table 6 and

$$
\begin{aligned}
\sum_{k=1}^{\frac{N-1}{2}} \sigma_{5}^{*}(k) \sigma_{5}^{*}(N-k)= & \sum_{k \equiv 0,1(\bmod 4)}^{\frac{N-1}{2}} \sigma_{5}^{*}(k) \sigma_{5}^{*}(N-k)+\sum_{k \equiv 2,7(\bmod 8)}^{\frac{N-1}{2}} \sigma_{5}^{*}(k) \sigma_{5}^{*}(N-k) \\
& +\sum_{k \equiv 3,6(\bmod 8)}^{\frac{N-1}{2}} \sigma_{5}^{*}(k) \sigma_{5}^{*}(N-k) .
\end{aligned}
$$

Table $6 \boldsymbol{k}$ and $N-k$ by $\bmod 8$

\begin{tabular}{lllllllll}
\hline$k(\bmod 8)$ & 0 & 1 & 2 & 3 & 4 & 5 & 6 & 7 \\
$N-k(\bmod 8)$ & 1 & 0 & 7 & 6 & 5 & 4 & 3 & 2 \\
\hline
\end{tabular}


If $n \equiv 7(\bmod 8)$, then there exists a prime $p \equiv 7(\bmod 8)$ satisfying $p^{2 i-1} \mid n$ and $p^{2 i} \nmid n$. Thus

$$
\begin{aligned}
\sigma_{5}^{*}(n) & =\sigma_{5}^{*}\left(\frac{n}{p^{2 i-1}}\right) \sigma_{5}^{*}\left(p^{2 i-1}\right) \\
& =\sigma_{5}^{*}\left(\frac{n}{p^{2 i-1}}\right)\left(1+p^{5}+p^{5 \cdot 2}+\cdots+p^{5(2 i-1)}\right) \\
& \equiv 0 \quad(\bmod 8) .
\end{aligned}
$$

Therefore, we obtain

$$
\begin{aligned}
\sigma_{5}^{*}(8 k+2) \sigma_{5}^{*}(8 k+7) & \equiv 2^{5} \sigma_{5}^{*}(4 k+1) \sigma_{5}^{*}(8 k+7) \\
& \equiv 0 \quad\left(\bmod 2^{8}\right) .
\end{aligned}
$$

Arguing in a similar manner as in (18), we get

$$
\sigma_{5}^{*}(8 k+3) \equiv 0 \quad(\bmod 4)
$$

and

$$
\sigma_{5}^{*}(4 k+3) \equiv 0 \quad(\bmod 4)
$$

Therefore, we obtain

$$
\begin{aligned}
\sigma_{5}^{*}(8 k+3) \sigma_{5}^{*}(8 k+6) & \equiv 2^{5} \sigma_{5}^{*}(8 k+3) \sigma_{5}^{*}(4 k+3) \\
& \equiv 0 \quad\left(\bmod 2^{9}\right)
\end{aligned}
$$

Using $\sigma_{5}^{*}(4 l)=2^{10} \sigma_{5}^{*}(l),(18)$, and Table 6 , we get

$$
\sum_{\substack{k=1 \\ k=0,1(\bmod 4)}}^{\frac{N-1}{2}} \sigma_{5}^{*}(k) \sigma_{5}^{*}(N-k) \equiv 0 \quad\left(\bmod 2^{10}\right)
$$

and

$$
\sum_{\substack{k=1 \\ k \equiv 2,3(\bmod 4)}}^{\frac{N-1}{2}} \sigma_{5}^{*}(k) \sigma_{5}^{*}(N-k) \equiv 0 \quad\left(\bmod 2^{8}\right) .
$$

Therefore, by (16), (20), and (21), we have

$$
\tau(N) \equiv \sigma_{11}(N) \quad(\bmod 1,415,168)
$$

Remark 5.4 The same result (c) of Theorem 5.3 has been obtained by Lahiri by a different approach considering the Eisenstein series and so on (see [4, (11.1)-(11.40)], [19, p.28]).

Corollary 5.5 Suppose that $N=176,896 a+b$ is a prime number with $(176,896, b)=1$. Then $\tau(N) \neq 0$ for $b \neq-1$. 
Proof Using Mathematica 8.0, we first find $b$ with $1 \leq b<176,896$ satisfying $(176,896$, $b)=1$. Then if $N=176,896 a+b$ is a prime number, then we find that by Theorem 5.3 (c), using Mathematica 8.0,

$$
\tau(N) \equiv \sigma_{11}^{*}(N) \equiv b^{11}+1 \not \equiv 0 \quad(\bmod 176,896)
$$

except for $b=-1$. Thus the corollary follows.

Example 5.6 Some values of $b(N), N \sigma_{5}(N), \sigma_{7}(N), \tau(N)$, and $\sigma_{11}(N)$ are listed in Table 7 .

Corollary 5.7 Let $N \in \mathbb{N}$. Then we have Table 8 .

In particular, if $N$ is odd, then we have Table 9.

Table 7 Some values of $b(N), N \sigma_{5}(N), \sigma_{7}(N), \tau(N)$, and $\sigma_{11}(N)$

\begin{tabular}{lrrrrrr}
\hline $\boldsymbol{N}$ & $\mathbf{1}$ & $\mathbf{3}$ & $\mathbf{5}$ & $\mathbf{7}$ & \multicolumn{2}{c}{$\mathbf{9}$} \\
\hline$b(N)$ & 1 & 12 & -210 & 1,016 & $-2,043$ \\
$N \sigma_{5}(N)$ & 1 & 732 & 15,630 & 117,656 & 533,637 \\
$\sigma_{7}(N)$ & 1 & 2,188 & 78,126 & 823,544 & $4,785,157$ \\
$\tau(N)$ & 1 & 252 & 4,830 & $-16,744$ & $-113,643$ \\
$\sigma_{11}(N)$ & 1 & 177,148 & $48,828,126$ & $1,977,326,744$ & $31,381,236,757$ \\
\hline
\end{tabular}

Table 8 Some convolution formulas

\begin{tabular}{ll}
\hline Convolution sum & Convolution formula \\
\hline$\sum_{k=1}^{N} \sigma_{1}(2 k-1) \sigma_{5}(2 N-(2 k-1))$ & $\frac{1}{408}\left\{768 \sigma_{7}^{*}(N)+5 b(2 N)-320 b(N)\right\}$ \\
$\sum_{k=1}^{N} \sigma_{3}(2 k-1) \sigma_{3}(2 N-(2 k-1))$ & $\frac{1}{136}\left\{64 \sigma_{7}^{*}(N)-b(2 N)+64 b(N)\right\}$ \\
$\sum_{k=1}^{N} \sigma_{1}(2 k-1) \sigma_{7}(2 N-(2 k-1))$ & $\frac{1}{496}\left\{2,176 \sigma_{9}^{*}(N)+224 d(2 N)-57,344 /(N)\right.$ \\
& $+7 c(2 N)-1,792 c(N)\}$ \\
$\sum_{k=1}^{N} \sigma_{3}(2 k-1) \sigma_{5}(2 N-(2 k-1))$ & $\frac{1}{496}\left\{256 \sigma_{9}^{*}(N)-32 d(2 N)+8,192 /(N)\right.$ \\
$\sum_{k=1}^{N} \sigma_{5}(2 k-1) \sigma_{5}(2 N-(2 k-1))$ & $\frac{1}{2,764}\left\{1,024 \sigma_{11}^{*}(N)-\tau(2 N)+1,716 \tau(N)\right.$ \\
$\sum_{k=1}^{N} \sigma_{1}(2 k-1) \sigma_{9}(2 N-(2 k-1))$ & $\frac{1}{27,640}\left\{317,4408,608 \tau\left(\frac{N}{2}\right)\right\}$ \\
& $\left.-280,656 \tau(N)-112,115,712 \tau\left(\frac{N}{2}\right)\right\}$ \\
$\sum_{k=1}^{N} \sigma_{3}(2 k-1) \sigma_{7}(2 N-(2 k-1))$ & $\frac{1}{22,112}\left\{17,408 \sigma_{11}^{*}(N)-17 \tau(2 N)+4,296 \tau(N)\right.$ \\
& $\left.+13,426,688 \tau\left(\frac{N}{2}\right)\right\}$ \\
\hline
\end{tabular}

Table 9 Some convolution formulas for odd $N$

\begin{tabular}{ll}
\hline Convolution sum & Convolution formula \\
\hline$\sum_{k=1}^{N} \sigma_{1}(2 k-1) \sigma_{5}(2 N-(2 k-1))$ & $\frac{1}{17}\left\{32 \sigma_{7}(N)-15 b(N)\right\}$ \\
$\sum_{k=1}^{N} \sigma_{3}(2 k-1) \sigma_{3}(2 N-(2 k-1))$ & $\frac{1}{17}\left\{8 \sigma_{7}(N)+9 b(N)\right\}$ \\
$\sum_{k=1}^{N} \sigma_{1}(2 k-1) \sigma_{7}(2 N-(2 k-1))$ & $\frac{1}{496}\left\{2,176 \sigma_{9}(N)+224 d(2 N)-57,344 /(N)+7 c(2 N)-1,792 c(N)\right\}$ \\
$\sum_{k=1}^{N} \sigma_{3}(2 k-1) \sigma_{5}(2 N-(2 k-1))$ & $\frac{1}{496}\left\{256 \sigma_{9}(N)-32 d(2 N)+8,192 /(N)-c(2 N)+256 c(N)\right\}$ \\
$\sum_{k=1}^{N} \sigma_{5}(2 k-1) \sigma_{5}(2 N-(2 k-1))$ & $\frac{1}{691}\left\{256 \sigma_{11}(N)+435 \tau(N)\right\}$ \\
$\sum_{k=1}^{N} \sigma_{1}(2 k-1) \sigma_{9}(2 N-(2 k-1))$ & $\frac{1}{691}\left\{7,936 \sigma_{11}(N)-7,245 \tau(N)\right\}$ \\
$\sum_{k=1}^{N} \sigma_{3}(2 k-1) \sigma_{7}(2 N-(2 k-1))$ & $\frac{1}{691}\left\{544 \sigma_{11}(N)+147 \tau(N)\right\}$ \\
\hline
\end{tabular}


Proof Since the proofs for the convolution sums are similar, we only prove $\sum_{k=1}^{N} \sigma_{1}(2 k-$ 1) $\sigma_{5}(2 N-(2 k-1))$. We write $\sum_{k=1}^{2 N-1} \sigma_{1}^{*}(k) \sigma_{5}^{*}(2 N-k)$ as follows:

$$
\begin{aligned}
& \sum_{k=1}^{2 N-1} \sigma_{1}^{*}(k) \sigma_{5}^{*}(2 N-k) \\
& \quad=\sum_{k=1}^{N} \sigma_{1}^{*}(2 k-1) \sigma_{5}^{*}(2 N-(2 k-1))+\sum_{k=1}^{N-1} \sigma_{1}^{*}(2 k) \sigma_{5}^{*}(2 N-2 k) \\
& \quad=\sum_{k=1}^{N} \sigma_{1}^{*}(2 k-1) \sigma_{5}^{*}(2 N-(2 k-1))+2^{6} \sum_{k=1}^{N-1} \sigma_{1}^{*}(k) \sigma_{5}^{*}(N-k)
\end{aligned}
$$

by (11). Also since $2 k-1$ and $2 N-(2 k-1)$ are odd, we find that

$$
\sum_{k=1}^{N} \sigma_{1}^{*}(2 k-1) \sigma_{5}^{*}(2 N-(2 k-1))=\sum_{k=1}^{N} \sigma_{1}(2 k-1) \sigma_{5}(2 N-(2 k-1)) .
$$

So, we obtain the formula for $\sum_{k=1}^{N} \sigma_{1}(2 k-1) \sigma_{5}(2 N-(2 k-1))$ by $(22)$ and Table 5 . In particular, if $N$ is odd, then $b(2 N)=b(2) b(N)$, because $b(N)$ is multiplicative. Therefore the proof is complete.

By eliminating $b(N), c(N)$, and $l(N)$ in Table 5 , we can obtain the following example.

Example 5.8 Let $N \in \mathbb{N}$. Then for $k=2,3$, 4, we have

$$
\sum_{s=0}^{k-1}\left(\begin{array}{c}
2 k \\
2 s+1
\end{array}\right)\left(\sum_{m=1}^{N-1} \sigma_{2 k-2 s-1}^{*}(m) \sigma_{2 s+1}^{*}(N-m)\right)=\frac{1}{2}\left\{\sigma_{2 k+1}^{*}(N)-N \sigma_{2 k-1}^{*}(N)\right\} .
$$

For $k=2$, we refer to Eq. (1).

Now we present some convolution formulas in Table 10.

\section{Certain combinatorial convolution sum}

The four basic theta functions are defined below following the notation of Whittaker and Watson [20, p.464]. Let $\tau \in \mathbb{C}$ be such that $\operatorname{Im}(\tau)>0$. Set $q=e^{\pi i \tau}$ so that $|q|<1$. For $z \in \mathbb{C}$, we define (as in [17])

$$
\begin{aligned}
& \theta_{1}(z, q):=2 \sum_{N=1}^{\infty}(-1)^{N-1} q^{\frac{(2 N-1)^{2}}{4}} \sin (2 N-1) z \\
& \theta_{2}(z, q):=2 \sum_{N=1}^{\infty} q^{\frac{(2 N-1)^{2}}{4}} \cos (2 N-1) z \\
& \theta_{3}(z, q):=1+2 \sum_{N=1}^{\infty} q^{N^{2}} \cos 2 N z \\
& \theta_{4}(z, q):=1+2 \sum_{N=1}^{\infty}(-1)^{N} q^{N^{2}} \cos 2 N z .
\end{aligned}
$$


Table 10 Some convolution formulas

\begin{tabular}{|c|c|}
\hline Convolution sum & Convolution formula \\
\hline$\sum_{k=1}^{N} \sigma_{1}(2 k) \sigma_{5}(2 N-2 k)$ & $\begin{array}{l}\frac{1}{8,568}\left[4\left\{85 \sigma_{7}(2 N)-4,032 \sigma_{7}^{*}(N)\right\}\right. \\
\quad-357(4 N-1) \sigma_{5}(2 N)+17 \sigma_{1}(2 N) \\
\quad-105\{b(2 N)-64 b(N)\}]\end{array}$ \\
\hline$\sum_{k=1}^{N} \sigma_{3}(2 k) \sigma_{3}(2 N-2 k)$ & $\begin{array}{l}\frac{1}{2,040}\left\{17 \sigma_{7}(2 N)-960 \sigma_{7}^{*}(N)\right. \\
\left.\quad-17 \sigma_{3}(2 N)+15 b(2 N)-960 b(N)\right\}\end{array}$ \\
\hline$\sum_{k=1}^{N} \sigma_{1}(2 k) \sigma_{7}(2 N-2 k)$ & $\begin{array}{l}\frac{1}{14,880}\left\{341 \sigma_{9}(2 N)-65,280 \sigma_{9}^{*}(N)\right. \\
\quad-620(3 N-1) \sigma_{7}(2 N)-31 \sigma_{1}(2 N) \\
\quad-6,720 /(2 N)+1,720,320 /(N) \\
\quad-210 c(2 N)+53,760 c(N)\}\end{array}$ \\
\hline$\sum_{k=1}^{N} \sigma_{3}(2 k) \sigma_{5}(2 N-2 k)$ & $\begin{array}{l}\frac{1}{156,240}\left\{341 \sigma_{9}(2 N)-80,640 \sigma_{9}^{*}(N)\right. \\
\quad-651 \sigma_{5}(2 N)+310 \sigma_{3}(2 N) \\
\quad+10,080 /(2 N)-25,80,480 /(N) \\
\quad+315 c(2 N)-80,640 c(N)\}\end{array}$ \\
\hline$\sum_{k=1}^{N} \sigma_{5}(2 k) \sigma_{5}(2 N-2 k)$ & $\begin{array}{l}\frac{1}{174,132}\left\{65 \sigma_{11}(2 N)-64,512 \sigma_{11}^{*}(N)\right. \\
\quad+691 \sigma_{5}(2 N)-693 \tau(2 N) \\
\left.\quad-108,108 \tau(N)+44,642,304 \tau\left(\frac{N}{2}\right)\right\}\end{array}$ \\
\hline$\sum_{k=1}^{N} \sigma_{1}(2 k) \sigma_{9}(2 N-2 k)$ & $\begin{array}{l}\frac{1}{912,120}\left[3\left\{4,550 \sigma_{11}(2 N)-3,491,840 \sigma_{11}^{*}(N)\right\}\right. \\
\quad-7,601(12 N-5) \sigma_{9}(2 N)+3,455 \sigma_{1}(2 N) \\
\quad+3\{-7,359 \tau(2 N)+3,087,216 \tau(N) \\
\left.\left.\quad+1,233,272,832 \tau\left(\frac{N}{2}\right)\right\}\right]\end{array}$ \\
\hline$\sum_{k=1}^{N} \sigma_{3}(2 k) \sigma_{7}(2 N-2 k)$ & $\begin{array}{l}\frac{1}{331,680}\left[3\left\{91 \sigma_{11}(2 N)-87,040 \sigma_{11}^{*}(N)\right\}\right. \\
\quad-1,382 \sigma_{7}(2 N)-691 \sigma_{3}(2 N) \\
\quad+3\{685 \tau(2 N)-21,480 \tau(N) \\
\left.\left.\quad-67,133,440 \tau\left(\frac{N}{2}\right)\right\}\right]\end{array}$ \\
\hline
\end{tabular}

Jacobi (see [21, 22]) proved that

$$
\frac{\theta_{2}(0, q) \theta_{3}(0, q) \theta_{1}(z, q)}{\theta_{4}(z, q)}=4 \sum_{m=0}^{\infty} \frac{q^{\frac{2 m+1}{2}}}{1-q^{2 m+1}} \sin (2 m+1) z
$$

and

$$
\frac{\theta_{2}^{2}(0, q) \theta_{3}^{2}(0, q) \theta_{1}^{2}(z, q)}{\theta_{4}^{2}(z, q)}=8 \sum_{m=0}^{\infty} \frac{m q^{m}}{1-q^{2 m}}(1-\cos 2 m z)
$$

From (23) and (24), we deduce that

$$
\sum_{m=1}^{\infty} \frac{m q^{m}}{1-q^{2 m}}(1-\cos 2 m z)=2\left(\sum_{m=0}^{\infty} \frac{q^{\frac{2 m+1}{2}}}{1-q^{2 m+1}} \sin (2 m+1) z\right)^{2}
$$

Equating coefficients of $q^{N}(N \in \mathbb{N})$ on the left- and right-hand sides of (25) (see [17, p.16]), then we obtain the arithmetical equality involving the trigonometric functions

$$
\sum_{\substack{m \in \mathbb{N} \\ m \mid N \\ \frac{N}{m} \text { odd }}} m(1-\cos 2 m z)=\sum_{\substack{(a, b, x, y) \in \mathbb{N}^{4} \\ a x+b y=2 N \\ a, b, x, y \text { odd }}}(\cos (a-b) z-\cos (a+b) z) .
$$

If we expand each cosine in powers of $z$ using

$$
1-\cos z=\sum_{k=1}^{\infty}(-1)^{k-1} \frac{z^{2 k}}{2 k !}
$$


and equate coefficients of $\frac{z^{2 k}}{2 k !}(k \in \mathbb{N})$, then we obtain

$$
\sum_{\substack{m \in \mathbb{N} \\ m \mid N \\ \frac{N}{m} \text { odd }}} 2^{2 k} m^{2 k+1}=\sum_{\substack{(a, b, x, y) \in \mathbb{N}^{4} \\ a x+b y=2 N \\ a, b, x, y \text { odd }}}\left((a+b)^{2 k}-(a-b)^{2 k}\right), \quad k, N \in \mathbb{N} .
$$

A generalized Besge formula due to Liouville is as follows.

Proposition 6.1 (See [17, Theorem 12.3]) Let $k \in \mathbb{N}$ and $N \in \mathbb{N}$, where $k, N \geq 2$. Then we have

$$
\begin{aligned}
& \sum_{s=0}^{k-1}\left(\begin{array}{c}
2 k \\
2 s+1
\end{array}\right)\left(\sum_{m=1}^{N-1} \sigma_{2 k-2 s-1}(m) \sigma_{2 s+1}(N-m)\right) \\
& \quad=\frac{2 k+3}{4 k+2} \sigma_{2 k+1}(N)+\left(\frac{k}{6}-N\right) \sigma_{2 k-1}(N)+\frac{1}{2 k+1} \sum_{j=2}^{k}\left(\begin{array}{c}
2 k+1 \\
2 j
\end{array}\right) B_{2 j} \sigma_{2 k+1-2 j}(N),
\end{aligned}
$$

where $B_{2 j}$ is the $2 j$ th Bernoulli number.

Let $\sigma_{s, o o}(N):=\sum_{\substack{d \mid N \\ d \\ \frac{n}{d} \text { odd }}} d^{s}$. Now we prove the following lemma.

Lemma 6.2 We have

$$
\sum_{s=0}^{k-1}\left(\begin{array}{c}
2 k \\
2 s+1
\end{array}\right) \sum_{m=1}^{2 N-1} \sigma_{2 k-2 s-1, o o}(m) \sigma_{2 s+1, o o}(2 N-m)=\frac{1}{4} \sigma_{2 k+1}^{*}(2 N) .
$$

Proof First we consider

$$
\sum_{\substack{(a, b, x, y) \in \mathbb{N}^{4}, a x+b y=2 N \\ a \equiv b \equiv x \equiv y \equiv 1 \bmod 2}}\left((a+b)^{2 k}-(a-b)^{2 k}\right)
$$

$$
\begin{aligned}
& =\sum_{\substack{(a, b, x, y) \in \mathbb{N}^{4}, a x+b y=2 N, a, b, x, y \text { odd }}}\left(\sum_{r=0}^{2 k}\left(\begin{array}{c}
2 k \\
r
\end{array}\right) a^{2 k-r} b^{r}-\sum_{r=0}^{2 k}\left(\begin{array}{c}
2 k \\
r
\end{array}\right)(-1)^{r} a^{2 k-r} b^{r}\right) \\
& =2 \sum_{\substack{(a, b, x, y) \in \mathbb{N}^{4}, a x+b y=2 N, a, b, x, y \text { odd }}} \sum_{r=0,}^{2 k}\left(\begin{array}{c}
2 k \\
r
\end{array}\right) a^{2 k-r} b^{r}=2 \sum_{s=0}^{k-1}\left(\begin{array}{c}
2 k \\
2 s+1
\end{array}\right) \sum_{\substack{(a, b, x, y) \in \mathbb{N}^{4}, a x+b y=2 N, a, b, x, y \text { odd }}} a^{2 k-2 s-1} b^{2 s+1} \\
& =2 \sum_{s=0}^{k-1}\left(\begin{array}{c}
2 k \\
2 s+1
\end{array}\right) \sum_{m=1}^{2 N-1}\left(\sum_{\substack{a \mid m, a \text { odd, } \\
\frac{m}{a} \text { odd }}} a^{2 k-2 s-1}\right)\left(\sum_{\substack{b \mid 2 N-m, b \text { odd, } \\
\frac{2 N-m}{b} \text { odd }}} b^{2 s+1}\right) \\
& =2 \sum_{s=0}^{k-1}\left(\begin{array}{c}
2 k \\
2 s+1
\end{array}\right) \sum_{m=1}^{2 N-1} \sigma_{2 k-2 s-1, o o}(m) \sigma_{2 s+1, o o}(2 N-m) .
\end{aligned}
$$


Now the right-hand side of (27) is

$$
\begin{aligned}
& \sum_{\substack{d \in \mathbb{N}, d \mid N, d \nmid \frac{N}{2}}} 2^{2 k} d^{2 k+1}=2^{2 k}\left\{\sum_{d \mid N} d^{2 k+1}-\sum_{d \mid \frac{N}{2}} d^{2 k+1}\right\} \\
& \quad=2^{2 k}\left\{\sigma_{2 k+1}(N)-\sigma_{2 k+1}\left(\frac{N}{2}\right)\right\}=2^{2 k} \sigma_{2 k+1}^{*}(N)=\frac{1}{2} \sigma_{2 k+1}^{*}(2 N)
\end{aligned}
$$

by (11). Therefore we obtain

$$
\sum_{s=0}^{k-1}\left(\begin{array}{c}
2 k \\
2 s+1
\end{array}\right) \sum_{m=1}^{N-1} \sigma_{2 k-2 s-1, o o}(m) \sigma_{2 s+1, o o}(N-m)=\frac{1}{4} \sigma_{2 k+1}^{*}(N)
$$

As a consequence of Proposition 6.1 and Lemma 6.2, we have the following.

\section{Theorem 6.3}

$$
\begin{aligned}
\sum_{s=0}^{k-1}\left(\begin{array}{c}
2 k \\
2 s+1
\end{array}\right) \sum_{m=1}^{N-1} \sigma_{2 k-2 s-1}(2 m) \sigma_{2 s+1}(2 N-2 m) \\
=\frac{2 k+3}{4 k+2} \sigma_{2 k+1}(2 N)-\frac{1}{4} \sigma_{2 k+1}^{*}(2 N)+\left(\frac{k}{6}-2 N\right) \sigma_{2 k-1}(2 N) \\
\quad+\frac{1}{2 k+1} \sum_{j=2}^{k}\left(\begin{array}{c}
2 k+1 \\
2 j
\end{array}\right) B_{2 j} \sigma_{2 k+1-2 j}(2 N),
\end{aligned}
$$

where $B_{2 j}$ is the 2jth Bernoulli number.

Proof From Proposition 6.1, we get

$$
\begin{gathered}
\sum_{s=0}^{k-1}\left(\begin{array}{c}
2 k \\
2 s+1
\end{array}\right)\left(\sum_{m=1}^{2 N-1} \sigma_{2 k-2 s-1}(m) \sigma_{2 s+1}(2 N-m)\right) \\
=\frac{2 k+3}{4 k+2} \sigma_{2 k+1}(2 N)+\left(\frac{k}{6}-2 N\right) \sigma_{2 k-1}(2 N) \\
+\frac{1}{2 k+1} \sum_{j=2}^{k}\left(\begin{array}{c}
2 k+1 \\
2 j
\end{array}\right) B_{2 j} \sigma_{2 k+1-2 j}(2 N)
\end{gathered}
$$

by replacing $N \rightarrow 2 N$. From Lemma 6.2 we know that

$$
\begin{gathered}
\sum_{s=0}^{k-1}\left(\begin{array}{c}
2 k \\
2 s+1
\end{array}\right) \sum_{m=1}^{2 N-1} \sigma_{2 k-2 s-1, o o}(m) \sigma_{2 s+1, o o}(2 N-m)=\frac{1}{4} \sigma_{2 k+1}^{*}(2 N) \\
=\sum_{s=0}^{k-1}\left(\begin{array}{c}
2 k \\
2 s+1
\end{array}\right) \sum_{m=1}^{N} \sigma_{2 k-2 s-1}(2 m-1) \sigma_{2 s+1}(2 N-2 m+1) .
\end{gathered}
$$

By subtracting (29) from (28), the theorem follows. 
Remark 6.4 It is well known that

$$
B_{0}=1, \quad B_{1}=-\frac{1}{2}, \quad B_{2}=\frac{1}{6}, \quad B_{2 j+1}=0 \quad(j \geq 1), \ldots
$$

In Proposition 6.1, taking $N=1$, we obtain

$$
\begin{aligned}
0= & \frac{2 k+3}{4 k+2}+\left(\frac{k}{6}-1\right)+\frac{1}{2 k+1} \sum_{j=2}^{k}\left(\begin{array}{c}
2 k+1 \\
2 j
\end{array}\right) B_{2 j} \\
= & \left(\frac{1}{2 k+1}+\frac{1}{2}\right)+\left(\frac{k}{6}-1\right)+\frac{1}{2 k+1} \sum_{j=2}^{k}\left(\begin{array}{c}
2 k+1 \\
2 j
\end{array}\right) B_{2 j} \\
= & \frac{1}{2 k+1}\left(\begin{array}{c}
2 k+1 \\
0
\end{array}\right) B_{0}+\frac{1}{2 k+1}\left(\begin{array}{c}
2 k+1 \\
1
\end{array}\right) B_{1}+\frac{1}{2 k+1}\left(\begin{array}{c}
2 k+1 \\
2
\end{array}\right) B_{2} \\
& +\frac{1}{2 k+1} \sum_{j=3}^{2 k}\left(\begin{array}{c}
2 k+1 \\
j
\end{array}\right) B_{j}
\end{aligned}
$$

and this implies the well-known identity involving the Bernoulli numbers $B_{j}$

$$
\frac{1}{2 k+1} \sum_{j=0}^{2 k}\left(\begin{array}{c}
2 k+1 \\
j
\end{array}\right) B_{j}=0 .
$$

In Theorem 6.3, taking $N=1$, we obtain

$$
\begin{aligned}
0= & \left(\frac{1}{2 k+1}+\frac{1}{2}\right)\left(1+2^{2 k+1}\right)+\left(\frac{k}{6}-1-1\right)\left(1+2^{2 k-1}\right)-\frac{1}{4} \cdot 2^{2 k+1} \\
& +\frac{1}{2 k+1} \sum_{j=2}^{k}\left(\begin{array}{c}
2 k+1 \\
2 j
\end{array}\right) B_{2 k+1-2 j} \cdot\left(1+2^{2 k+1-2 j}\right) .
\end{aligned}
$$

Then we have

$$
\begin{aligned}
0= & \left(\frac{1}{2 k+1}+\frac{1}{2}\right)+\left(\frac{k}{6}-1\right)+\frac{1}{2 k+1} \sum_{j=2}^{k}\left(\begin{array}{c}
2 k+1 \\
2 j
\end{array}\right) B_{2 k+1-2 j} \\
& +\frac{1}{2 k+1} 2^{2 k+1}+2^{2 k}-2^{2 k-1}+\frac{k}{6} \cdot 2^{2 k-1} \\
& +\frac{1}{2 k+1} \sum_{j=2}^{k}\left(\begin{array}{c}
2 k+1 \\
2 j
\end{array}\right) B_{2 k+1-2 j} 2^{2 k+1-2 j}-1-2^{2 k-1}-2^{2 k-1} .
\end{aligned}
$$

Using (30) and (31), we get

$$
\begin{aligned}
0= & \frac{1}{2 k+1}\left(\begin{array}{c}
2 k+1 \\
0
\end{array}\right) 2^{2 k+1}-\frac{1}{2 k+1}\left(\begin{array}{c}
2 k+1 \\
1
\end{array}\right) 2^{2 k+1-1}\left(-\frac{1}{2}\right) \\
& +\frac{1}{2 k+1}\left(\begin{array}{c}
2 k+1 \\
2
\end{array}\right) 2^{2 k+1-2}+\frac{1}{2 k+1} \sum_{j=3}^{2 k}\left(\begin{array}{c}
2 k+1 \\
j
\end{array}\right)(-1)^{j} B_{2 k+1-2 j} 2^{2 k+1-2 j},
\end{aligned}
$$


and hence again we obtain a more general relation between Bernoulli numbers and the Faulhaber sum

$$
\frac{1}{2 k+1} \sum_{j=0}^{2 k}\left(\begin{array}{c}
2 k+1 \\
j
\end{array}\right)(-1)^{j} B_{2 k+1-j} 2^{2 k+1-j}=1+2^{2 k}=S_{2 k}(3) .
$$

See, for example, [23]

\section{Appendix}

The values of $a(N), b(N), c(N), l(N)$, and $\tau(N)$ (for $(1 \leq N \leq 45)$ ) are given in Tables 11, $12,13,14$, and 15 respectively. We first write the corresponding product expression into a $q$-series sum of a finite number of terms (i.e., $q$-series sum of $N$ number of terms) with an error which tends to zero as $N \rightarrow \infty$. Then we use Mathematica 8.0 to compare the corresponding coefficients up to $N \leq 45$.

Evaluation of certain convolution sums: We have Table 16.

Table $11 a(N)$ for $N(1 \leq N \leq 45)$

\begin{tabular}{|c|c|c|c|c|c|}
\hline$N$ & $a(N)$ & $\boldsymbol{N}$ & $a(N)$ & $\boldsymbol{N}$ & $a(N)$ \\
\hline 1 & 1 & 16 & 16 & 31 & -88 \\
\hline 2 & -2 & 17 & -126 & 32 & -32 \\
\hline 3 & -3 & 18 & -18 & 33 & -36 \\
\hline 4 & 4 & 19 & 20 & 34 & 252 \\
\hline 5 & 6 & 20 & 24 & 35 & -96 \\
\hline 6 & 6 & 21 & 48 & 36 & 36 \\
\hline 7 & -16 & 22 & -24 & 37 & 254 \\
\hline 8 & -8 & 23 & 168 & 38 & -40 \\
\hline 9 & 9 & 24 & 24 & 39 & -114 \\
\hline 10 & -12 & 25 & -89 & 40 & -48 \\
\hline 11 & 12 & 26 & -76 & 41 & 42 \\
\hline 12 & -12 & 27 & -27 & 42 & -96 \\
\hline 13 & 38 & 28 & -64 & 43 & -52 \\
\hline 14 & 32 & 29 & 30 & 44 & 48 \\
\hline 15 & -18 & 30 & 36 & 45 & 54 \\
\hline
\end{tabular}

Table $12 b(N)$ for $N(1 \leq N \leq 45)$

\begin{tabular}{|c|c|c|c|c|c|}
\hline$N$ & $b(N)$ & $N$ & $b(N)$ & $N$ & $b(N)$ \\
\hline 1 & 1 & 16 & 4,096 & 31 & 227,552 \\
\hline 2 & -8 & 17 & 14,706 & 32 & $-32,768$ \\
\hline 3 & 12 & 18 & 16,344 & 33 & 13,104 \\
\hline 4 & 64 & 19 & $-39,940$ & 34 & $-117,648$ \\
\hline 5 & -210 & 20 & $-13,440$ & 35 & $-213,360$ \\
\hline 6 & -96 & 21 & 12,192 & 36 & $-130,752$ \\
\hline 7 & 1,016 & 22 & $-8,736$ & 37 & 160,526 \\
\hline 8 & -512 & 23 & 68,712 & 38 & 319,520 \\
\hline 9 & $-2,043$ & 24 & $-6,144$ & 39 & 16,584 \\
\hline 10 & 1,680 & 25 & $-34,025$ & 40 & 107,520 \\
\hline 11 & 1,092 & 26 & $-11,056$ & 41 & 10,842 \\
\hline 12 & 768 & 27 & $-50,760$ & 42 & $-97,536$ \\
\hline 13 & 1,382 & 28 & 65,024 & 43 & $-630,748$ \\
\hline 14 & $-8,128$ & 29 & $-102,570$ & 44 & 69,888 \\
\hline 15 & $-2,520$ & 30 & 20,160 & 45 & 429,030 \\
\hline
\end{tabular}


Table $13 c(N)$ for $N(1 \leq N \leq 45)$

\begin{tabular}{rr}
\hline $\boldsymbol{N}$ & $\mathbf{c ( N )}$ \\
\hline 1 & 1 \\
2 & -16 \\
3 & 100 \\
4 & -256 \\
5 & -154 \\
6 & 2,496 \\
7 & $-4,536$ \\
8 & $-4,096$ \\
9 & 23,085 \\
10 & $-13,920$ \\
11 & $-38,996$ \\
12 & 39,936 \\
13 & 37,806 \\
14 & 15,232 \\
15 & $-146,472$ \\
\hline
\end{tabular}

\begin{tabular}{lr}
\hline $\boldsymbol{N}$ & \multicolumn{1}{c}{$\boldsymbol{c ( \boldsymbol { N } )}$} \\
\hline 16 & $-65,536$ \\
17 & 311,442 \\
18 & $-74,448$ \\
19 & 128,244 \\
20 & $-222,720$ \\
21 & $-912,352$ \\
22 & 898,368 \\
23 & 767,192 \\
24 & 638,976 \\
25 & $-1,405,121$ \\
26 & $-2,849,504$ \\
27 & $2,699,496$ \\
28 & 243,712 \\
29 & $1,659,454$ \\
30 & $2,171,520$ \\
\hline
\end{tabular}

\begin{tabular}{lr}
\hline $\boldsymbol{N}$ & $\boldsymbol{c ( \boldsymbol { N } )}$ \\
\hline 31 & $-5,370,592$ \\
32 & $-1,048,576$ \\
33 & $-1,704,144$ \\
34 & $3,962,592$ \\
35 & $2,533,552$ \\
36 & $-1,191,168$ \\
37 & $8,258,694$ \\
38 & $-5,046,080$ \\
39 & $-14,176,264$ \\
40 & $-3,563,520$ \\
41 & $12,739,178$ \\
42 & $-2,376,192$ \\
43 & $11,055,308$ \\
44 & $14,373,888$ \\
45 & $-12,992,274$ \\
\hline
\end{tabular}

Table $14 I(N)$ for $N(1 \leq N \leq 45)$

\begin{tabular}{rr}
\hline $\boldsymbol{N}$ & $\boldsymbol{I}(\boldsymbol{N})$ \\
\hline 1 & 0 \\
2 & 1 \\
3 & -8 \\
4 & 16 \\
5 & 32 \\
6 & -156 \\
7 & 112 \\
8 & 256 \\
9 & -576 \\
10 & 870 \\
11 & -536 \\
12 & $-2,496$ \\
13 & 4,384 \\
14 & -952 \\
15 & 336 \\
\hline
\end{tabular}

\begin{tabular}{lr}
\hline $\boldsymbol{N}$ & \multicolumn{1}{c}{$\boldsymbol{I ( \boldsymbol { N } )}$} \\
\hline 16 & 4,096 \\
17 & $-17,472$ \\
18 & 4,653 \\
19 & 5,848 \\
20 & 13,920 \\
21 & 33,152 \\
22 & $-56,148$ \\
23 & $-17,584$ \\
24 & $-39,936$ \\
25 & 6,528 \\
26 & 178,094 \\
27 & $-11,088$ \\
28 & $-15,232$ \\
29 & $-171,872$ \\
30 & $-135,720$ \\
\hline
\end{tabular}

\begin{tabular}{rr}
\hline $\boldsymbol{N}$ & \multicolumn{1}{c}{$\boldsymbol{I ( \boldsymbol { N } )}$} \\
\hline 31 & 126,912 \\
32 & 65,536 \\
33 & 326,976 \\
34 & $-247,662$ \\
35 & $-105,056$ \\
36 & 74,448 \\
37 & $-123,488$ \\
38 & 315,380 \\
39 & $-425,200$ \\
40 & 222,720 \\
41 & $-350,848$ \\
42 & 148,512 \\
43 & 706,728 \\
44 & $-898,368$ \\
45 & 532,512 \\
\hline
\end{tabular}

Table $15 \tau(N)$ for $N(1 \leq N \leq 45)$

\begin{tabular}{|c|c|c|c|}
\hline$N$ & $\tau(N)$ & $N$ & $\tau(N)$ \\
\hline 1 & 1 & 16 & 987,136 \\
\hline 2 & -24 & 17 & $-6,905,934$ \\
\hline 3 & 252 & 18 & $2,727,432$ \\
\hline 4 & $-1,472$ & 19 & $10,661,420$ \\
\hline 5 & 4,830 & 20 & $-7,109,760$ \\
\hline 6 & $-6,048$ & 21 & $-4,219,488$ \\
\hline 7 & $-16,744$ & 22 & $-12,830,688$ \\
\hline 8 & 84,480 & 23 & $18,643,272$ \\
\hline 9 & $-113,643$ & 24 & $21,288,960$ \\
\hline 10 & $-115,920$ & 25 & $-25,499,225$ \\
\hline 11 & 534,612 & 26 & $13,865,712$ \\
\hline 12 & $-370,944$ & 27 & $-73,279,080$ \\
\hline 13 & $-577,738$ & 28 & $24,647,168$ \\
\hline 14 & 401,856 & 29 & $128,406,630$ \\
\hline 15 & $1,217,160$ & 30 & $-29,211,840$ \\
\hline
\end{tabular}

\begin{tabular}{lr}
\hline $\boldsymbol{N}$ & $\boldsymbol{\tau}(\boldsymbol{N})$ \\
\hline 31 & $-52,843,168$ \\
32 & $-196,706,304$ \\
33 & $134,722,224$ \\
34 & $165,742,416$ \\
35 & $-80,873,520$ \\
36 & $167,282,496$ \\
37 & $-182,213,314$ \\
38 & $-255,874,080$ \\
39 & $-145,589,976$ \\
40 & $408,038,400$ \\
41 & $308,120,442$ \\
42 & $101,267,712$ \\
43 & $-17,125,708$ \\
44 & $-786,948,864$ \\
45 & $-548,895,690$ \\
\hline
\end{tabular}


Table 16 Some convolution formulas

\begin{tabular}{|c|c|c|}
\hline Convolution sum & Convolution formulas & Reference \\
\hline$\sum_{k=1}^{N-1} \sigma(k) \sigma_{5}(N-k)$ & $\begin{array}{l}\frac{1}{504}\left\{20 \sigma_{7}(N)+(21\right. \\
\left.\quad-42 N) \sigma_{5}(N)+\sigma(N)\right\}\end{array}$ & {$[3,(3.18)]$} \\
\hline$\sum_{k=1}^{N-1} \sigma_{3}(k) \sigma_{3}(N-k)$ & $\frac{1}{120}\left\{\sigma_{7}(N)-\sigma_{3}(N)\right\}$ & {$[3,(3.17)]$} \\
\hline$\sum_{k=1}^{N-1} \sigma(k) \sigma_{7}(N-k)$ & $\begin{array}{l}\frac{1}{480}\left\{11 \sigma_{9}(N)+(20\right. \\
\left.\quad-30 N) \sigma_{7}(N)-\sigma(N)\right\}\end{array}$ & {$[3,(3.28)]$} \\
\hline$\sum_{k=1}^{N-1} \sigma_{3}(k) \sigma_{5}(N-k)$ & $\begin{array}{l}\frac{1}{5,040}\left\{11 \sigma_{9}(N)-21 \sigma_{5}(N)\right. \\
\left.\quad+10 \sigma_{3}(N)\right\}\end{array}$ & {$[3,(3.27)]$} \\
\hline$\sum_{k=1}^{N-1} \sigma_{5}(k) \sigma_{5}(N-k)$ & $\begin{array}{l}\frac{3}{691}\left\{\frac{65}{756} \sigma_{11}(N)+\frac{691}{756} \sigma_{5}(N)\right. \\
\quad-\tau(N)\}\end{array}$ & {$[4,(21)]$} \\
\hline$\sum_{k=1}^{N-1} \sigma_{1}(k) \sigma_{9}(N-k)$ & $\begin{array}{l}\frac{455}{30,404} \sigma_{11}(N)-\frac{N}{20} \sigma_{9}(N) \\
\quad+\frac{1}{24} \sigma_{9}(N)+\frac{1}{264} \sigma_{1}(N) \\
\quad-\frac{36}{3,455} \tau(N)\end{array}$ & {$[4,(19)]$} \\
\hline$\sum_{k=1}^{N-1} \sigma_{3}(k) \sigma_{7}(N-k)$ & $\begin{array}{l}\frac{91}{110,560} \sigma_{11}(N)-\frac{1}{240} \sigma_{7}(N) \\
\quad-\frac{1}{480} \sigma_{3}(N)+\frac{15}{2,764} \tau(N)\end{array}$ & {$[4,(20)]$} \\
\hline$\sum_{k<N / 2} \sigma_{5}(k) \sigma_{1}(N-2 k)$ & $\begin{array}{l}\frac{1}{2,142} \sigma_{7}(N)+\frac{2}{51} \sigma_{7}\left(\frac{N}{2}\right) \\
\quad+\frac{1-2 N}{24} \sigma_{5}\left(\frac{N}{2}\right) \\
\quad+\frac{1}{504} \sigma_{1}(N)-\frac{1}{408} b(N)\end{array}$ & {$[24$, Theorem 5.2] } \\
\hline$\sum_{k<N / 2} \sigma_{1}(k) \sigma_{5}(N-2 k)$ & $\begin{array}{l}\frac{1}{102} \sigma_{7}(N)+\frac{32}{1,071} \sigma_{7}\left(\frac{N}{2}\right) \\
\quad+\frac{1-N}{24} \sigma_{5}(N) \\
\quad+\frac{1}{504} \sigma_{1}\left(\frac{N}{2}\right)-\frac{1}{102} b(N)\end{array}$ & {$[24$, Theorem 5.2] } \\
\hline$\sum_{k<N / 2} \sigma_{3}(k) \sigma_{3}(N-2 k)$ & $\begin{array}{l}\frac{1}{2,040} \sigma_{7}(N)+\frac{2}{255} \sigma_{7}\left(\frac{N}{2}\right) \\
\quad-\frac{1}{240} \sigma_{3}(N) \\
\quad-\frac{1}{240} \sigma_{3}\left(\frac{N}{2}\right)+\frac{1}{272} b(N)\end{array}$ & {$[24$, Theorem 5.2] } \\
\hline$\sum_{k<N / 2} \sigma_{7}(k) \sigma_{1}(N-2 k)$ & $\begin{array}{l}\frac{1}{14,880} \sigma_{9}(N)+\frac{17}{744} \sigma_{9}\left(\frac{N}{2}\right) \\
\quad+\frac{2-3 N}{48} \sigma_{7}\left(\frac{N}{2}\right) \\
\quad-\frac{1}{480} \sigma_{1}(N)+\frac{1}{496} c(N) \\
\quad+\frac{2}{31} /(N)\end{array}$ & {$[24$, Theorem 6.2] } \\
\hline$\sum_{k<N / 2} \sigma_{1}(k) \sigma_{7}(N-2 k)$ & $\begin{array}{l}\frac{17}{2,976} \sigma_{9}(N)+\frac{8}{465} \sigma_{9}\left(\frac{N}{2}\right) \\
\quad+\frac{4-3 N}{96} \sigma_{7}(N) \\
\quad-\frac{1}{480} \sigma_{1}\left(\frac{N}{2}\right)-\frac{1}{62} c(N) \\
\quad-\frac{16}{31} /(N)\end{array}$ & {$[24$, Theorem 6.2] } \\
\hline$\sum_{k<N / 2} \sigma_{5}(k) \sigma_{3}(N-2 k)$ & $\begin{array}{l}\frac{1}{31,248} \sigma_{9}(N)+\frac{1}{465} \sigma_{9}\left(\frac{N}{2}\right) \\
\quad-\frac{1}{240} \sigma_{5}\left(\frac{N}{2}\right)+\frac{1}{504} \sigma_{3}(N) \\
\quad-\frac{1}{496} c(N)-\frac{2}{31} l(N)\end{array}$ & {$[24$, Theorem 6.2] } \\
\hline$\sum_{k<N / 2} \sigma_{3}(k) \sigma_{5}(N-2 k)$ & $\begin{array}{l}\frac{1}{7,440} \sigma_{9}(N)+\frac{4}{1,953} \sigma_{9}\left(\frac{N}{2}\right) \\
\quad-\frac{1}{240} \sigma_{5}(N)+\frac{1}{504} \sigma_{3}\left(\frac{N}{2}\right) \\
\quad+\frac{1}{248} c(N)+\frac{4}{31} /(N)\end{array}$ & {$[24$, Theorem 6.2] } \\
\hline$\sum_{k<N / 2} \sigma_{5}(k) \sigma_{5}(N-2 k)$ & $\begin{array}{l}\frac{1}{174,132} \sigma_{11}(N)+\frac{16}{43,533} \sigma_{11}\left(\frac{N}{2}\right) \\
\quad+\frac{1}{504} \sigma_{5}(N)+\frac{1}{504} \sigma_{5}\left(\frac{N}{2}\right) \\
\quad-\frac{11}{5,528} \tau(N)-\frac{88}{691} \tau\left(\frac{N}{2}\right)\end{array}$ & {$[24$, Theorem 7.2] } \\
\hline$\sum_{k<N / 2} \sigma_{9}(k) \sigma_{1}(N-2 k)$ & $\begin{array}{l}\frac{1}{91,212} \sigma_{11}(N)+\frac{31}{2,073} \sigma_{11}\left(\frac{N}{2}\right) \\
\quad+\frac{5-6 N}{120} \sigma_{9}\left(\frac{N}{2}\right)+\frac{1}{264} \sigma_{1}(N) \\
\quad-\frac{21}{5,528} \tau(N)-\frac{282}{3,455} \tau\left(\frac{N}{2}\right)\end{array}$ & {$[24$, Theorem 7.2] } \\
\hline$\sum_{k<N / 2} \sigma_{1}(k) \sigma_{9}(N-2 k)$ & $\begin{array}{l}\frac{31}{8,292} \sigma_{11}(N)+\frac{256}{22,803} \sigma_{11}\left(\frac{N}{2}\right) \\
\quad+\frac{5-3 N}{120} \sigma_{9}(N)+\frac{1}{266} \sigma_{1}\left(\frac{N}{2}\right) \\
\quad-\frac{141}{6,910} \tau(N)-\frac{2,688}{691} \tau\left(\frac{N}{2}\right)\end{array}$ & {$[24$, Theorem 7.2] } \\
\hline$\sum_{k<N / 2} \sigma_{7}(k) \sigma_{3}(N-2 k)$ & $\begin{array}{l}\frac{1}{331,680} \sigma_{11}(N)+\frac{17}{20,730} \sigma_{11}\left(\frac{N}{2}\right) \\
\quad-\frac{1}{240} \sigma_{7}\left(\frac{N}{2}\right)-\frac{1}{480} \sigma_{3}(N) \\
\quad+\frac{23}{11,056} \tau(N)+\frac{91}{1,382} \tau\left(\frac{N}{2}\right)\end{array}$ & {$[24$, Theorem 7.2] } \\
\hline$\sum_{k<N / 2} \sigma_{3}(k) \sigma_{7}(N-2 k)$ & $\begin{array}{l}\frac{17}{331,680} \sigma_{11}(N)+\frac{8}{10,365} \sigma_{11}\left(\frac{N}{2}\right) \\
\quad-\frac{1}{240} \sigma_{7}(N)-\frac{1}{480} \sigma_{3}\left(\frac{N}{2}\right) \\
\quad+\frac{91}{22,112} \tau(N)+\frac{368}{691} \tau\left(\frac{N}{2}\right)\end{array}$ & {$[24$, Theorem 7.2] } \\
\hline
\end{tabular}




\section{Competing interests}

The authors declare that they have no competing interests.

\section{Authors' contributions}

All authors contributed equally to the manuscript and typed, read and approved the final manuscript.

\section{Author details}

${ }^{1}$ National Institute for Mathematical Sciences, Yuseong-daero 1689-gil, Yuseong-gu, Daejeon, 305-811, South Korea.

${ }^{2}$ Department of Mathematics and Institute of Pure and Applied Mathematics, Chonbuk National University, Chonju, Chonbuk 561-756, Korea. ${ }^{3}$ Current address: National Institute for Mathematical Sciences, Yuseong-daero 1689-gil, Yuseong-gu, Daejeon, 305-811, South Korea. ${ }^{4}$ Permanent address: School of Mathematics, Tata Institute of Fundamental Research, Homi Bhabha Road, Mumbai, 400005, India.

\section{Acknowledgements}

The author AS wishes to thank the National Institute for Mathematical Sciences (NIMS), Daejeon, Republic of Korea for its warm hospitality and generous support.

\section{Received: 20 February 2013 Accepted: 23 April 2013 Published: 6 May 2013}

\section{References}

1. Ramanujan, S: On certain arithmetical functions. Trans. Cambridge Philos. Soc. 22, 159-184 (1916)

2. Ramanujan, S: Collected Papers. Am. Math. Soc., Providence (2000)

3. Huard, JG, Ou, ZM, Spearman, BK, Williams, KS: Elementary evaluation of certain convolution sums involving divisor functions. In: Number Theory for the Millennium, vol. II, pp. 229-274 (2002)

4. Lahiri, DB: On Ramanujan's function $\tau(n)$ and the divisor function $\sigma_{k}$, I. Bull. Calcutta Math. Soc. 38, $193-206$ (1946)

5. Glaisher, JWL: On certain sums of products of quantities depending upon the divisors of a number. Mess. Math. 15, $1-20(1885)$

6. Levitt, J: On a Problem of Ramanujan. M. Phil thesis, University of Nottingham (1978)

7. Melfi, G: On Some Modular Identities, Number Theory, pp. 371-382. de Gruyter, Berlin (1998)

8. Glaisher, JWL: On the square of the series in which the coefficients are the sums of the divisors of the exponents. Mess. Math. 14, 156-163 (1884)

9. Glaisher, JWL: Expressions for the five powers of the series in which the coefficients are the sums of the divisors of the exponents. Mess. Math. 15, 33-36 (1885)

10. Hahn, H: Convolution sums of some functions on divisors. Rocky Mt. J. Math. 37, 1593-1622 (2007)

11. Lahiri, DB: On Ramanujan's function $\tau(n)$ and the divisor function $\sigma_{k}$, II. Bull. Calcutta Math. Soc. 39, 33-52 (1947)

12. Alaca, S, Williams, KS: Evaluation of the convolution sums $\sum_{1+6 m=n} \sigma(I) \sigma(m)$ and $\sum_{21+3 m=n} \sigma(I) \sigma(m)$. J. Number Theory $124,491-510$ (2007)

13. Simsek, Y: Elliptic analogue of the Hardy sums related to elliptic Bernoulli functions. Gen. Math. 15, 3-23 (2007)

14. Ozden, H, Cangul, IN, Simsek, Y: Multivariate interpolation functions of higher-order $q$-Euler numbers and their applications. Abstr. Appl. Anal. 2008, Article ID 390857 (2008)

15. Kim, M-S, Lee, JH: On sums of the extended q-Euler numbers. J. Math. Anal. Appl. 397, 522-528 (2013)

16. Kim, A, Kim, D, Seo, G: Convolution sum $\sum_{k<N / 3} \sigma_{1}\left(3^{m} k\right) \sigma_{1}\left(2^{n}(N-3 k)\right)$. Honam Math. J. 34, 519-531 (2012)

17. Williams, KS: Number Theory in the Spirit of Liouville. London Mathematical Society, Student Texts, vol. 76. Cambridge University Press, Cambridge (2011)

18. Kim, D, Kim, A, Park, H: Congruences of the Weierstrass $\wp(x)$ and $\wp^{\prime \prime}(x)\left(x=\frac{1}{2}, \frac{\tau}{2}, \frac{\tau+1}{2}\right)$-functions on divisors. Bull. Korean Math. Soc. 50, 241-261 (2013)

19. Berndt, BC, Ono, K: Ramanujan's unpublished manuscript on the partition and tau functions with proofs and commentary. Lotharingien de Combinatoire, vol. 42,63 pp. (1999)

20. Whittaker, ET, Watson, GN: A Course of Modern Analysis. Cambridge University Press, Cambridge (1963)

21. Jacobi, CGJ: Fundamenta Nova Theoriae Functionum Ellipticarum. Sumptibus Fratrum Bornträger; reprinted in C. G. J. Jacobi, Gesammelte Werke, vol. 1, pp. 49-239. Reimer, Berlin (1881-1891)

22. Jacobi, CGJ: Gesammelte Werke, vols. I-VIII. Chelsea, New York (1969)

23. http://en.wikipedia.org/wiki/Bernoulli_number

24. Cheng, N, Williams, KS: Evaluation of some convolution sums involving the sum of divisors functions. Yokohama Math. J. 52, 39-57 (2005)

doi:10.1186/1029-242X-2013-225

Cite this article as: Kim et al.: Bernoulli numbers, convolution sums and congruences of coefficients for certain generating functions. Journal of Inequalities and Applications 2013 2013:225. 